Алгебра и анализ

Том. 17 (2005), вып. 2
St. Petersburg Math. J.

Vol. 17 (2006), No. 2, Pages 343-375

S 1061-0022(06)00908-3

Article electronically published on February 20, 2006

\title{
A TROPICAL APPROACH TO ENUMERATIVE GEOMETRY
}

\author{
E. SHUSTIN
}

\begin{abstract}
A detailed algebraic-geometric background is presented for the tropical approach to enumeration of singular curves on toric surfaces, which consists of reducing the enumeration of algebraic curves to that of non-Archimedean amoebas, the images of algebraic curves by a real-valued non-Archimedean valuation. This idea was proposed by Kontsevich and recently realized by Mikhalkin, who enumerated the nodal curves on toric surfaces. The main technical tools are a refined tropicalization of one-parametric equisingular families of curves and the patchworking construction of singular algebraic curves. The case of curves with a cusp and the case of real nodal curves are also treated.
\end{abstract}

\section{$\S 1$. INTRODUCTION}

The rapid development of tropical algebraic geometry over recent years has led to interesting applications to enumerative geometry of singular algebraic curves, proposed by Kontsevich 16. The first result in this direction was obtained by Mikhalkin [18, who counted the curves with a given number of nodes on toric surfaces via lattice paths in convex lattice polygons. Our main goal in the present paper is to explain this breakthrough result, notably the link between algebraic curves and non-Archimedean amoebas, which is the core of the tropical approach to enumerative geometry. Our point of view is purely algebraic-geometric and differs from Mikhalkin's method, which is based on symplectic geometry techniques. Briefly speaking, we count equisingular families of curves over a punctured disk. The tropicalization procedure extends such families to the central point, and these tropical limits are basically encoded by non-Archimedean amoebas. In its turn, the patchworking construction restores an equisingular family out of the central fiber.

Tropicalization. Let $\Delta \subset \mathbb{R}^{2}$ be a convex lattice polygon, and let $\operatorname{Tor}_{\mathbb{K}}(\Delta)$ be the toric surface associated with the polygon $\Delta$ and defined over an algebraically closed field $\mathbb{K}$ of characteristic zero. We denote by $\Lambda_{\mathbb{K}}(\Delta)$ the tautological linear system on $\operatorname{Tor}_{\mathbb{K}}(\Delta)$ generated by the monomials $x^{i} y^{j},(i, j) \in \Delta \cap \mathbb{Z}^{2}$. We would like to count the $n$-nodal curves belonging to $\Lambda_{\mathbb{K}}(\Delta)$ and passing through $r=\operatorname{dim} \Lambda_{\mathbb{K}}(\Delta)-n=\left|\Delta \cap \mathbb{Z}^{2}\right|-1-n$ generic points in $\operatorname{Tor}_{\mathbb{K}}(\Delta)$, i.e., we want to find the degree of the so-called Severi variety $\Sigma_{\Delta}\left(n A_{1}\right)$. Let $\mathbb{K}$ be the field of convergent Puiseux series over $\mathbb{C}$, i.e., power series of the form $b(t)=\sum_{\tau \in R} c_{\tau} t^{\tau}$, where $R \subset \mathbb{Q}$ is contained in an arithmetic progression bounded from below, and $\sum_{\tau \in R}\left|c_{\tau}\right| t^{\tau}<\infty$ for sufficiently small positive $t$. The field $\mathbb{K}$ is equipped with a non-Archimedean valuation $\operatorname{Val}(b)=-\min \left\{\tau \in R: c_{\tau} \neq 0\right\}$, which

2000 Mathematics Subject Classification. Primary 14H15; Secondary 12J25, 14H20, 14M25, 14N10.

Key words and phrases. Singular curves, toric surfaces, tropicalization.

The author was supported by the German-Israeli Foundation for Research and Development (grant no. G-616-15.6/99), by the Hermann Minkowski Minerva Center for Geometry at Tel Aviv University, and by the Bessel research award from the Alexander von Humboldt Foundation. 
takes $\mathbb{K}^{*}$ onto $\mathbb{Q}$ and satisfies

$$
\operatorname{Val}(a b)=\operatorname{Val}(a)+\operatorname{Val}(b), \quad \operatorname{Val}(a+b) \leq \max \{\operatorname{Val}(a), \operatorname{Val}(b)\}, \quad a, b \in \mathbb{K}^{*} .
$$

A curve $C \in \Lambda_{\mathbb{K}}(\Delta)$ with $n$ nodes is given by a polynomial

$$
f(x, y)=\sum_{(i, j) \in \Delta \cap \mathbb{Z}^{2}} a_{i j}(t) x^{i} y^{j}, \quad a_{i j}(t) \in \mathbb{K} .
$$

Without loss of generality we can assume that all exponents of $t$ in $a_{i j}(t)$ are integral, and thus, polynomial (1.0.1) determines an analytic surface $X$ in $Y=\operatorname{Tor}(\Delta) \times(D \backslash\{0\}),{ }^{1}$ where $D$ is a small disk in $\mathbb{C}$ centered at 0 , and $X$ is such that the fibers $X_{t}$ are complex algebraic curves that belong to the linear system $\Lambda(\Delta)$ on the surface $\operatorname{Tor}(\Delta)$ and have $n$ nodes (cf. Lemma 2.3 and Subsection 2.2).

With the pair $\left(\operatorname{Tor}_{\mathbb{K}}(\Delta), C\right)$ we associate a certain limit of the family $\left(Y_{t}, X_{t}\right)$ as $t \rightarrow 0$, where $Y_{t}=\operatorname{Tor}(\Delta) \times\{t\} \subset Y$. The result $\left(Y_{0}, X_{0}\right)$ of this operation is called the tropicalization (or dequantization) of the pair $\left(\operatorname{Tor}_{\mathbb{K}}(\Delta), C\right.$ ). Namely, the surface $Y_{0}$ splits into irreducible components $Y_{0,1}, \ldots, Y_{0, N}$, corresponding to a subdivision of $\Delta$ into convex lattice polygons, and this subdivision is dual to the non-Archimedean amoeba $A_{f} \subset \mathbb{R}^{2}$ of the polynomial $f$ that passes through the points $\left(\operatorname{Val}\left(x_{i}\right), \operatorname{Val}\left(y_{i}\right)\right) \in \mathbb{R}^{2} . \operatorname{Next}$ we define a refinement of a tropicalization as the tropicalization of the corresponding polynomial $f$ after a certain change of coordinates. This refinement corresponds to blow-ups of the threefold $\bar{Y}=Y \cup Y_{0}$ at some singular points of $X_{0}$ or along multiple components of $X_{0}$; it extends $Y_{0}$ by adding exceptional divisors and extends the curve $X_{0}$ by adding new components, which we call deformation patterns.

We show that the refined tropicalizations $\left(Y_{0}, X_{0}\right)$ of the $n$-nodal curves $C \in \operatorname{Tor}_{\mathbb{K}}(\Delta)$ passing through $\left(x_{i}, y_{i}\right) \in\left(\mathbb{K}^{*}\right)^{2}, i=1, \ldots, r$, belong to a certain finite set $T$. Using our patchworking theorem, we decide how many $n$-nodal curves $C \in \operatorname{Tor}_{\mathbb{K}}(\Delta)$ passing through $\left(x_{i}, y_{i}\right) \in\left(\mathbb{K}^{*}\right)^{2}, i=1, \ldots, r$, arise from an element $\left(Y_{0}, X_{0}\right)$ of $T$; and thus, we obtain $\operatorname{deg} \Sigma_{\Delta}\left(n A_{1}\right)$ as the sum of weights of elements of $T$. In fact, we seek the family $X_{t}$ in the form (1.0.1) where the tropicalization provides some initial terms in the coefficients $a_{i j}(t)$.

Here we do not touch the merely combinatorial problem of counting the elements of $T$. Mikhalkin [18] did this in a nice elementary way, by tracing non-Archimedean amoebas through points on a straight line and attaching the dual subdivisions to lattice paths in $\Delta$.

Also, we would like to note that the tropical approach can be applied to counting curves with other singularities, and here we demonstrate this for a relatively simple case of curves with an ordinary cusp. The main difficulty in the general case is to describe the possible tropicalizations, whereas the patchworking Theorem 5 applies to curves with arbitrary singularities.

Furthermore, if the given points in $\left(\mathbb{K}^{*}\right)^{2}$ are invariant with respect to complex conjugation, one can count the real tropicalizations, and, thus, the real singular curves passing through given points. We discuss this in $\S 6$ in connection with the Welschinger invariant [35].

Patchworking construction. In 1979-1980, O. Viro [30, 31, 32, 33, invented a patchworking construction for real nonsingular algebraic hypersurfaces. It should be mentioned that almost all known topological types of real nonsingular algebraic curves are realized in this way.

\footnotetext{
${ }^{1}$ From now on the symbol Tor $(*)$ will always mean a toric variety over $\mathbb{C}$.
} 
In general, the initial data of the construction consist of

- a one-parametric flat family $F \rightarrow(\mathbb{F}, 0)$ of algebraic varieties $Y_{t}$ of dimension at least 2, with $\mathbb{F}=\mathbb{C}$ or $\mathbb{R}$, where $Y_{0}$ is assumed to be reduced (reducible), and $Y_{t}$, $t \neq 0$, irreducible;

- a line bundle $\mathcal{L}$ on $Y$;

- the zero locus $X_{0} \subset Y_{0}$ of some section $S$ of $\left.\mathcal{L}\right|_{Y_{0}}$, which is assumed to be a hypersurface in $Y_{0}$.

The construction extends $S$ up to a section of $\mathcal{L}$, whose zero locus $X \subset Y$ determines a family of hypersurfaces $X_{t} \subset Y_{t}$; the latter inherit some properties of $X_{0}$. In 30 , 31, 32, 33, $Y$ is a toric variety associated with a convex lattice polytope and fibered into toric hypersurfaces $Y_{t}, t>0$, which degenerate into the union of some divisors on $Y$ corresponding to facets of the polytope, and $X_{0}$ is a union of nonsingular real algebraic hypersurfaces. The real nonsingular hypersurfaces $X_{t} \subset Y_{t}, t \neq 0$, arise as a result of a topological gluing (patchworking) of the components of $X_{0}$.

In the early 1990s, the author suggested using the patchworking construction for tracing other properties of objects defined by polynomials, for example, prescribed singularities of algebraic hypersurfaces [22, 25, 26, critical points of polynomials 24, 25, singular points and limit cycles of planar polynomial vector fields [10], resultants of bivariate polynomials 23. For the case where $\operatorname{dim} Y=3, \operatorname{dim} Y_{t}=2, \operatorname{dim} X_{0}=1$, in [22, 25, 26], we always assumed that the components of the curve $X_{0}$ are reduced and meet the intersection lines of the components of the surface $Y_{0}$ transversally at their nonsingular points. The novelty of the patchworking theorem presented in this paper (Theorem 5 in Subsection 5.3) is that we allow $X_{0}$ to be nonreduced and to have singularities along $\operatorname{Sing}\left(Y_{0}\right)$.

In this connection we would like to note that, in [3] (see also [4]), a deformation $Y \rightarrow(\mathbb{C}, 0)$ of surfaces in $\mathbb{P}^{3}$ with reducible $Y_{0}$ was considered, where the components of $X_{0}$ are nodal curves tangent to the intersection lines of the components of $Y_{0}$. For example, Theorem 2.1 in $[3]$ claims that a point on the intersection line of two components of $Y_{0}$ at which nonsingular germs of the corresponding components of $X_{0}$ have contact of order $m$, gives rise to $m-1$ nodes of $X_{t} \subset Y_{t}, t \neq 0$, and the proof is based on a technically tricky result by Caporaso and Harris [1, Lemma 4.1] (see also [2, Lemma 2.8]). Our approach is to interpret this as a patchworking, i.e., a replacement of a neighborhood of a singular point by some algebraic curve, or more precisely, by an affine curve with Newton triangle $\{(0,0),(0,2),(m, 1)\}$ and with an arbitrary number $0 \leq k \leq m-1$ of nodes (cf. 22, Proposition 2.5]). Extensive development of this idea, covering a broad class of possible singularities, was done in [28]. However, the result of [28] is not sufficient, for example, for the patchworking nodal curves, as required in the enumeration problem.

Organization of the material. In $\S 2$ we provide preliminary information on nonArchimedean amoebas and tropicalizations of polynomials. $\$ 3$ contains Theorem 3 , which reduces enumeration of the nodal curves lying in toric surfaces and associated with convex lattice polygons to counting the nodal non-Archimedean amoebas that pass through the respective number of generic points in the real plane. In $\S 4$ we prove Theorem 4. which allows us to reduce the enumeration of curves with one cusp to the count of appropriate cuspidal non-Archimedean amoebas. In the proof of Theorems 3 and 4 we formulate explicit patchworking statements, which invert the tropicalization procedure, and which follow from the main patchworking Theorem 5 presented in $\$ 5$. Finally, in $\$ 6$ we demonstrate an application of our technique to computation of the Welschinger number for real nodal curves in toric surfaces. 

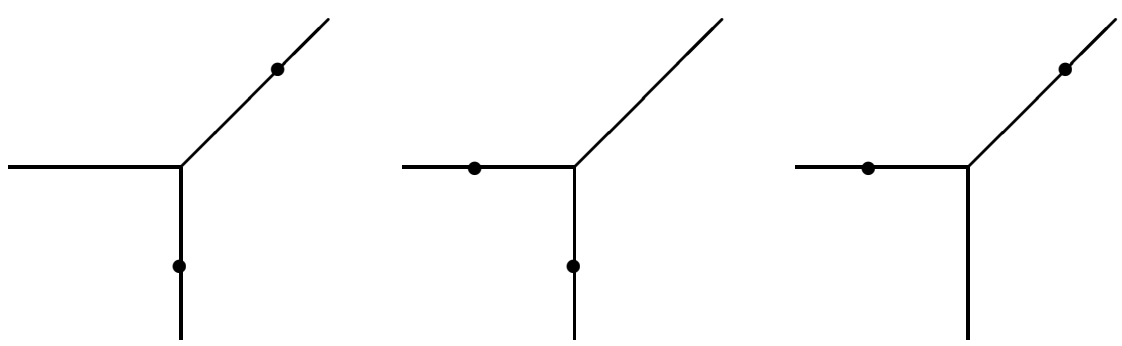

FiguRE 1. Plane amoebas of the first order

Acknowledgment. I am very grateful to G. Mikhalkin and I. Itenberg for useful discussions. Also, I wish to thank Universität Kaiserslautern for hospitality and excellent working conditions.

\section{§2. Non-ARChimedeAn AMOEBAS}

2.1. Preliminaries. Amoebas of complex algebraic hypersurfaces were introduced in [7] and studied further in [6, 8, 15, 16, 17, 20, 21]. We are interested in "non-Archimedean amoebas", i.e., those defined over fields with a non-Archimedean valuation [14, 16. The field $\mathbb{K}$ of convergent Puiseux series over $\mathbb{C}$ serves as an example. For a nonempty finite set $I \subset \mathbb{Z}^{k}$, let $F_{\mathbb{K}}(I)$ denote the set of Laurent polynomials

$$
f(\boldsymbol{z})=\sum_{\omega \in I} c_{\omega} \boldsymbol{z}^{\omega}, \quad \boldsymbol{z}=\left(z_{1}, \ldots, z_{k}\right), \quad c_{\omega} \in \mathbb{K}^{*}, \omega \in I .
$$

We put $Z_{f}=\{f=0\} \subset\left(\mathbb{K}^{*}\right)^{k}$ and define the amoeba $A_{f}$ of $f$ as the closure of the set $\operatorname{Val}\left(Z_{f}\right) \subset \mathbb{R}^{k}$, where $\operatorname{Val}\left(z_{1}, \ldots, z_{k}\right)=\left(\operatorname{Val}\left(z_{1}\right), \ldots, \operatorname{Val}\left(z_{n}\right)\right)$. The set of amoebas $A_{f}$, $f \in F_{\mathbb{K}}(I)$, is denoted by $\mathcal{A}(I)$. If $I$ is the set of all integral points in a convex lattice polygon $\Delta$, we write $\mathcal{A}(\Delta)$.

The following simple observation is due to Kapranov [12] (see also [27]).

Theorem 1. The amoeba $A_{f}$ coincides with the corner locus of the piecewise linear convex function

$$
N_{f}(\boldsymbol{x})=\max _{\omega \in I}\left(\omega \boldsymbol{x}+\operatorname{Val}\left(c_{\omega}\right)\right), \quad \boldsymbol{x} \in \mathbb{R}^{k} .
$$

(Here and in what follows, the product of vectors means the standard scalar product.)

The non-Archimedean amoebas unexpectedly reveal many properties common to algebraic varieties. For example [16], there is one and only one amoeba of a straight line through two generic points in the plane (see Figure 1). Similarly, there exists one and only one amoeba of a conic curve through five generic points in the plane. To introduce the reader to the subject, we extend this existence and uniqueness result to amoebas of polynomials in any number of variables and with arbitrary support.

Theorem 2. For any integers $k \geq 2, n \geq 1$, any finite set $I \subset \mathbb{Z}^{k}$ consisting of $n+1$ points, and any generic $n$-tuple $\left(\boldsymbol{x}_{1}, \ldots, \boldsymbol{x}_{n}\right) \in\left(\mathbb{Q}^{k}\right)^{n}$, there exists one and only one amoeba $A \in \mathcal{A}(I)$ passing through $\boldsymbol{x}_{1}, \ldots, \boldsymbol{x}_{n}$.

Proof. The existence part is trivial: simply take the amoeba of a polynomial $f \in F_{\mathbb{K}}(I)$ with $Z_{f}$ passing through any $n$-tuple $\left(w_{1}, \ldots, w_{n}\right) \in \operatorname{Val}^{-1}\left(\boldsymbol{x}_{1}, \ldots, \boldsymbol{x}_{n}\right) \subset\left(\left(\mathbb{K}^{*}\right)^{k}\right)^{n}$.

To prove uniqueness, we impose the following condition on $\boldsymbol{x}_{1}, \ldots, \boldsymbol{x}_{n}$. Consider the $(n \times(n+1))$-matrix $M$ whose $i$ th row entries are $\boldsymbol{x}_{i} \omega, \omega \in I$. Assume that all the 
sums of $n$ entries of $M$ taken one from each row and one from each but one column, are distinct. Clearly, this excludes a finite number of hyperplanes in $\left(\mathbb{R}^{k}\right)^{n}$. Given $n$ points $w_{1}, \ldots, w_{n} \in\left(\mathbb{K}^{*}\right)^{k}$, we can find the coefficients $c_{\omega}, \omega \in I$, of the polynomial $f \in F_{\mathbb{K}}(I)$ vanishing at these points as the $(n \times n)$-minors (with signs) of the $(n \times(n+1))$ matrix $N$ whose $i$ th row entries are $w_{i}^{\omega}, \omega \in I$. If $\operatorname{Val}\left(w_{1}, \ldots, w_{n}\right)=\left(\boldsymbol{x}_{1}, \ldots, \boldsymbol{x}_{n}\right)$, then $\operatorname{Val}\left(c_{\omega}\right)$ will be the maximal sum of $n$ entries of $M$ taken one from each row and one from each but the $\omega$ th column. Thus, $\operatorname{Val}\left(c_{\omega}\right)$ does not depend on the choice of $\left(w_{1}, \ldots, w_{n}\right)$ in $\operatorname{Val}^{-1}\left(\boldsymbol{x}_{1}, \ldots, \boldsymbol{x}_{n}\right)$; hence, by Theorem 1, all such polynomials produce the same amoeba.

2.2. Amoebas and subdivisions of the Newton polytope. Any polynomial $f \in$ $F_{\mathbb{K}}(I)$ allows us to define a subdivision of the Newton polytope $\Delta=\operatorname{conv}(I)$ into convex polytopes with vertices in $I$. Namely, we take the convex hull $\widehat{\Delta}$ of the set $\left\{\left(\omega,-\operatorname{Val}\left(c_{\omega}\right)\right) \in \mathbb{R}^{k+1}: \omega \in I\right\}$ and introduce the function

$$
\nu_{f}: \Delta \rightarrow \mathbb{R}, \quad \nu_{f}(\omega)=\min \{x:(\omega, x) \in \widehat{\Delta}\} .
$$

This is a convex piecewise linear function. Its linearity domains are convex polytopes with vertices in $I$, which form a subdivision $S_{f}$ of $\Delta$. The following result is easy to deduce, e.g., from the fact that the functions $N_{f}$ and $\nu_{f}$ are dual to each other with respect to the Legendre transformation.

Lemma 2.1. The subdivision $S_{f}$ of $\Delta$ is combinatorially dual to the pair $\left(\mathbb{R}^{k}, A_{f}\right)$.

Observe that, in general, the geometry of an amoeba $A \in \mathcal{A}(\Delta)$ determines the dual subdivision $S$ of $\Delta$ not uniquely, but up to a combinatorial isotopy in which all edges remain orthogonal to the corresponding edges of $A$, and vice versa. The combinatorially isotopic amoebas form a subset ${ }^{2}$ in $\mathcal{A}(I)$, whose dimension we call the rank of an amoeba (or the rank of a subdivision) and denote $\operatorname{rk}\left(A_{f}\right)=\operatorname{rk}\left(S_{f}\right)$.

Lemma 2.2. If $k=2$ and $S_{f}: \Delta=\Delta_{1} \cup \cdots \cup \Delta_{N}$, then

$$
\operatorname{rk}\left(S_{f}\right) \geq \operatorname{rk}_{v}\left(S_{f}\right) \stackrel{\text { def }}{=}\left|V\left(S_{f}\right)\right|-1-\sum_{i=1}^{N}\left(\left|V\left(\Delta_{i}\right)\right|-3\right),
$$

where $V\left(S_{f}\right)$ is the set of vertices of $S_{f}, V\left(\Delta_{i}\right)$ is the set of vertices of the polygon $\Delta_{i}$, $i=1, \ldots, N$. More precisely,

$$
\operatorname{rk}\left(S_{f}\right)=\operatorname{rk}_{v}\left(S_{f}\right)+d\left(S_{f}\right),
$$

where

- $d\left(S_{f}\right)=0$ if all the polygons $\Delta_{1}, \ldots, \Delta_{N}$ are triangles or parallelograms;

- otherwise, we have

$$
0 \leq 2 d\left(S_{f}\right) \leq \sum_{m \geq 2}\left((2 m-3) N_{2 m}-N_{2 m}^{\prime}\right)+\sum_{m \geq 2}(2 m-2) N_{2 m+1}-1,
$$

where $N_{m}, m \geq 3$, is the number of m-gons in $S_{f}$, and $N_{2 m}^{\prime}, m \geq 2$, is the number of $2 m$-gons in $S_{f}$, whose opposite edges are parallel.

Proof. Inequality (2.2.2) is obvious, because an $m$-valent vertex of $A_{f}$ imposes $m-3$ linear conditions on the planes forming the graph of $N_{f}$.

Assuming that all $\Delta_{1}, \ldots, \Delta_{N}$ are triangles or parallelograms, we show that the conditions imposed by the 4 -valent vertices of $A_{f}$ are independent. We take a vector $\bar{a} \in \mathbb{R}^{2}$ with an irrational slope and coorient each edge of any parallelogram so that the normal vector forms an acute angle with $\bar{a}$. This coorientation determines a partial ordering

${ }^{2}$ In fact, this subset is the interior of a convex polyhedron in $\mathcal{A}(I)$. 
on the set of parallelograms, and we complete this ordering somehow up to a linear ordering. Observe that each parallelogram has two neighboring edges cooriented outside. Altogether, this means that the coefficients of the linear conditions imposed by the 4 -valent vertices of $A_{f}$ can be arranged into a triangular matrix, and, hence, are independent, i.e., $d\left(S_{f}\right)=0$.

If $S_{f}$ contains polygons different from triangles and parallelograms, we define a linear ordering on the set of all non-triangles in the same way as above. Denote by $e_{-}\left(\Delta_{i}\right)$ (respectively, $e_{+}\left(\Delta_{i}\right)$ ) the number of edges of a polygon $\Delta_{i}$ cooriented outside (respectively, inside) $\Delta_{i}$. Passing inductively over the nontriangular polygons $\Delta_{i}$, each time we add at least $\min \left\{e_{-}\left(\Delta_{i}\right)-1,\left|V\left(\Delta_{i}\right)\right|-3\right\}$ new linear conditions independent of all the preceding ones. Thus,

$$
\begin{aligned}
d\left(S_{f}\right) & \leq \sum_{i=2}^{N}\left(\left|V\left(\Delta_{i}\right)\right|-3-\min \left\{e_{-}\left(\Delta_{i}\right)-1,\left|V\left(\Delta_{i}\right)\right|-3\right\}\right) \\
& =\sum_{i=2}^{N} \max \left\{\left|V\left(\Delta_{i}\right)\right|-e_{-}\left(\Delta_{i}\right)-2,0\right\},
\end{aligned}
$$

because, for the initial polygon $\Delta_{1}$, all $\left|V\left(\Delta_{1}\right)\right|-3$ imposed conditions are independent. Replacing $\bar{a}$ by $-\bar{a}$, we obtain

$$
d\left(S_{f}\right) \leq \sum_{i=1}^{N-1} \max \left\{\left|V\left(\Delta_{i}\right)\right|-e_{+}\left(\Delta_{i}\right)-2,0\right\} .
$$

Since

- the relations $1 \leq e_{-}\left(\Delta_{i}\right) \leq\left|V\left(\Delta_{i}\right)\right|-1$ and $e_{-}\left(\Delta_{i}\right)+e_{+}\left(\Delta_{i}\right)=\left|V\left(\Delta_{i}\right)\right|$ yield

$$
\begin{aligned}
& \max \left\{\left|V\left(\Delta_{i}\right)\right|-e_{-}\left(\Delta_{i}\right)-2,0\right\}+\max \left\{\left|V\left(\Delta_{i}\right)\right|-e_{+}\left(\Delta_{i}\right)-2,0\right\} \\
& \quad \leq\left|V\left(\Delta_{i}\right)\right|-3 ;
\end{aligned}
$$

- for a $2 m$-gon with parallel opposite edges we have

$$
\begin{aligned}
e_{-} & =e_{+}=m \\
& \Longrightarrow \max \left\{2 m-e_{-}-2,0\right\}+\max \left\{2 m-e_{+}-2,0\right\}=2 m-4,
\end{aligned}
$$

we get

$$
2 d\left(S_{f}\right) \leq \sum_{m \geq 2}\left((2 m-3) N_{2 m}-N_{2 m}^{\prime}\right)+\sum_{m \geq 2}(2 m-2) N_{2 m+1} .
$$

If among $\Delta_{1}, \ldots, \Delta_{N}$ there is a polygon $\Delta_{i}$ whose number of edges is odd and exceeds 3 , or a polygon with an even number of edges and a pair of nonparallel opposite sides, then $\bar{a}$ can be chosen so that $\min \left\{e_{-}\left(\Delta_{i}\right), e_{+}\left(\Delta_{i}\right)\right\} \geq 2$. Thus, the contribution of $\Delta_{i}$ to the bound for $2 d\left(S_{f}\right)$ will be $\left|V\left(\Delta_{i}\right)\right|-4$, which allows us to gain -1 on the right-hand side of (2.2.5), obtaining (2.2.4).

Finally, assume that all nontriangular polygons in $S_{f}$ have an even number of edges, that their opposite sides are parallel, and furthermore, that there is $\Delta_{i}$ with $\left|V\left(\Delta_{i}\right)\right|=$ $2 m \geq 6$. The union of the finite length edges of $A_{f}$ is the adjacency graph of $\Delta_{1}, \ldots, \Delta_{N}$. We take the vertex corresponding to $\Delta_{i}$, pick a generic point $O$ in a small neighborhood of this vertex, and orient each finite length edge of $A_{f}$ so that it form an acute angle with the radius vector from $O$ to the middle point of the chosen edge. Equipped with such an orientation, the adjacency graph has no oriented cycles, because the terminal point of any edge is farther from $O$ than the initial point. Thus, we obtain a partial ordering on $\Delta_{1}, \ldots, \Delta_{N}$ such that, for any $\Delta_{k}$ with an even number of edges, at least half of the edges are cooriented outside. Then we apply the preceding argument to estimate 
$d\left(S_{f}\right)$ and notice that the contribution of the initial polygon $\Delta_{i}$ to such a bound is zero, whereas on the right-hand side of (2.2.5) it is at least two. This completes the proof of (2.2.4).

2.3. Algebraic curves over $\mathbb{K}$ and $\mathbb{C}$ : general fiber and tropicalization. Let $\Delta \subset \mathbb{R}^{2}$ be a nondegenerate convex lattice polygon, and let $C \in \Lambda_{\mathbb{K}}(\Delta)$ be a curve with only isolated singularities, which is determined by a polynomial $f(x, y)$ as in (1.0.1). This curve gives rise to some complex algebraic curves.

First, evaluating the coefficients of $f(x, y)$ at small positive $t$ (or at complex nonzero $t$ close to zero if the exponents of $t$ in the coefficients $a_{i j}(t)$ of $f(x, y)$ are integral), we obtain a family of curves $C^{(t)} \in \Lambda(\Delta)$. The relationship between $C$ and $C^{(t)}$ is formulated in the following statement, in which by the topological type of an isolated singular point (over any algebraically closed field of characteristic zero) we mean a minimal resolution tree with given multiplicities of the point itself and of its infinitely near points, or equivalently, the number of local branches, their characteristic Puiseux exponents, and pairwise intersection multiplicities.

Lemma 2.3. The collection of topological types of the singular points of a reduced curve $C \in \Lambda_{\mathbb{K}}(\Delta)$ coincides with the collection of topological types of the singular points of a generic curve $C^{(t)} \in \Lambda(\Delta)$. A curve $C \in \Lambda_{\mathbb{K}}(\Delta)$ is reducible if and only if a generic curve $C^{(t)} \in \Lambda(\Delta)$ is reducible.

This immediately follows from the fact that, for a given linear system, the set of curves with singularities of prescribed topological types over any algebraically closed field of characteristic zero is determined by the same system of polynomial equalities and inequalities with integral coefficients. The same argument confirms the simultaneous reducibility of $C$ and $C^{(t)}$.

By shrinking the range of $t$ if necessary, we can arrange that the curve $C$ bears a one-parameter equisingular deformation of complex curves.

We shall also define certain limits of $C^{(t)}$ as $t \rightarrow 0$. Namely, let $\nu_{f}: \Delta \rightarrow \mathbb{R}$ be a convex function, and let $S_{f}$ be the corresponding subdivision $\Delta=\Delta_{1} \cup \cdots \cup \Delta_{N}$, as defined in the preceding subsection. The restriction $\left.\nu_{f}\right|_{\Delta_{i}}$ coincides with a linear (affine) function $\lambda_{i}: \Delta \rightarrow \mathbb{R}, \lambda_{i}(\boldsymbol{x})=\omega_{i} \boldsymbol{x}+\gamma_{i}, \omega_{i}=\left(\alpha_{1}, \alpha_{2}\right) \in \mathbb{R}^{2}, \gamma_{i} \in \mathbb{R}, i=1, \ldots, N$. Then the polynomial

$$
t^{-\gamma_{i}} f\left(z_{1} t^{-\alpha_{1}}, z_{2} t^{-\alpha_{2}}\right)=\sum_{\omega \in \Delta \cap \mathbb{Z}^{2}} \widetilde{c}_{\omega} z^{\omega}
$$

satisfies the following condition:

$$
\operatorname{Val}\left(\widetilde{c}_{\omega}\right) \begin{cases}=0 & \text { if } \omega \text { is a vertex of } \Delta_{i}, \\ \leq 0 & \text { if } \omega \in \Delta_{i}, \\ <0 & \text { if } \omega \notin \Delta_{i} .\end{cases}
$$

In other words, letting $t=0$ on the right-hand side of (2.3.6), we obtain a complex polynomial $f_{i}$ with Newton polygon $\Delta_{i}$; in its turn, this polynomial determines a complex curve $C_{i} \in \Lambda\left(\Delta_{i}\right), i=1, \ldots, N$. Notice that multiplying $f(x, y)$ by a constant from $\mathbb{K}^{*}$ does not change $S_{f}$ and $C_{1}, \ldots, C_{N}$, but adds a linear function to $\nu_{f}$. The collection $\left(\nu_{f}, S_{f} ; C_{1}, \ldots, C_{N}\right)$ is called the tropicalization (or dequantization) of the curve $C$ and is denoted by $\mathcal{T}(C)$. We also call the $f_{i}$ and $C_{i}$ the tropicalizations of the polynomial $f$ and the curve $C$ on the polygon $\Delta_{i}, 1 \leq i \leq N$.

By a change of parameter $t \mapsto t^{m}$, we can make all these exponents of $t$ integral and the function $\nu_{f}$ integral-valued at integral points. Introduce the polyhedron

$$
\widetilde{\Delta}=\left\{(\alpha, \beta, \gamma) \in \mathbb{R}^{3}:(\alpha, \beta) \in \Delta, \gamma \geq \nu_{f}(\alpha, \beta)\right\}
$$


It determines a toric variety $Y=\operatorname{Tor}(\widetilde{\Delta})$, which naturally fibers over $\mathbb{C}$ so that the fibers $Y_{t}$ over $t \neq 0$ are isomorphic to $\operatorname{Tor}(\Delta)$, and $Y_{0}$ is the union of the toric surfaces $\operatorname{Tor}\left(\widetilde{\Delta}_{i}\right)$, $i=1, \ldots, N$, where $\widetilde{\Delta}_{1}, \ldots, \widetilde{\Delta}_{N}$ are the faces of the graph of $\nu_{f}$. By the choice of $\nu_{f}$, we have $\operatorname{Tor}\left(\widetilde{\Delta}_{i}\right) \simeq \operatorname{Tor}\left(\Delta_{i}\right)$, and we shall simply write $Y_{0}=\bigcup_{i} \operatorname{Tor}\left(\Delta_{i}\right)$. Then the curve $C$ can be viewed as an analytic surface in a neighborhood of $Y_{0}$, which fibers into the complex curves $C^{(t)} \subset Y_{t} \simeq \operatorname{Tor}(\Delta)$ and whose closure intersects $Y_{0}$ along the curve $C^{(0)}$ that can be identified with $\bigcup_{i} C_{i} \subset \bigcup_{i} \operatorname{Tor}\left(\Delta_{i}\right)$. Passing if necessary to a finite cyclic covering ramified along $Y_{0}$, we can assume that $\operatorname{Tor}(\widetilde{\Delta})$ is nonsingular everywhere except, possibly, at finitely many points corresponding to vertices of $\widetilde{\Delta}$, and, moreover, the surfaces $\operatorname{Tor}\left(\Delta_{k}\right) \backslash \operatorname{Sing}(\operatorname{Tor}(\widetilde{\Delta})), k=1, \ldots, N$, are smooth and intersect transversally in $\operatorname{Tor}(\widetilde{\Delta}) \backslash \operatorname{Sing}(\operatorname{Tor}(\widetilde{\Delta}))$.

The singular points of the curves $C^{(t)}$ determine sections $s: D \backslash\{0\} \rightarrow \operatorname{Tor}(\widetilde{\Delta})$, where $D \subset \mathbb{C}$ is a small disk centered at 0 . The limit points $z=\lim _{t \rightarrow 0} s(t)$ are singular points of $C^{(0)}$. We say that such a point $z \in C^{(0)}$ bears the corresponding singular points of $C^{(t)}$. If $z \in C^{(0)}$ does not belong to the intersection lines $\bigcup_{i \neq j} \operatorname{Tor}\left(\Delta_{i} \cap \Delta_{j}\right)$ and bears only one singular point of $C^{(t)}$, which is topologically equivalent to $z$, we call $z$ a regular singular point; otherwise it is irregular. If $C^{(0)}$ has irregular singular points, we can define a refinement of the tropicalization in the following way: transform the polynomial $f(x, y)$ into $f(x+a, y+b)$ with $a, b \in \mathbb{K}$ in such a way that the irregular singular point of $C^{(0)}$ goes to the origin, and consider the tropicalization of the curve defined by the new polynomial $f(x+a, y+b)$. This provides additional information on the behavior of the singular points of $C^{(t)}$ that tend to irregular singular points of $C^{(0)}$, and corresponds, in a sense, to blowing up the threefold $Y$ at the irregular singular points of $C^{(0)}$ (cf. [28]).

\section{$\S 3$. Counting nodal CuRves}

3.1. Formulation of the result. Let $\Delta \subset \mathbb{R}^{2}$ be a nondegenerate lattice polygon that has integral points in its interior. It is well known that the number of nodes of an irreducible curve in $\Lambda_{\mathbb{K}}(\Delta)$ does not exceed $\left|\operatorname{Int}(\Delta) \cap \mathbb{Z}^{2}\right|$. For any positive integer $n \leq\left|\operatorname{Int}(\Delta) \cap \mathbb{Z}^{2}\right|$, we denote by $\Sigma_{\Delta}\left(n A_{1}\right)$ the set of reduced curves in $\Lambda_{\mathbb{K}}(\Delta)$ that have exactly $n$ nodes as their only singularities and are defined by polynomials with Newton polygon $\Delta$. This is a smooth quasiprojective subvariety of $\Lambda_{\mathbb{K}}(\Delta)$ (the so-called Severi variety) of codimension $n$, i.e., $\operatorname{dim} \Sigma_{\Delta}\left(n A_{1}\right)=r=\left|\Delta \cap \mathbb{Z}^{2}\right|-1-n$, because $\operatorname{dim} \Lambda_{\mathbb{K}}(\Delta)=\left|\Delta \cap \mathbb{Z}^{2}\right|-1$. Imposing the condition of passing through $r$ generic points in $\left(\mathbb{K}^{*}\right)^{2} \subset \operatorname{Tor}_{\mathbb{K}}(\Delta)$, we obtain a finite set of curves in $\Sigma_{\Delta}\left(n A_{1}\right)$; the cardinality of it is simply $\operatorname{deg} \Sigma_{\Delta}\left(n A_{1}\right)$.

Now we describe the amoebas that are projections of nodal curves passing through generic points in $\left(\mathbb{K}^{*}\right)^{2}$ and with distinct valuation projections to $\mathbb{R}^{2}$. An amoeba $A \in$ $\mathcal{A}(\Delta)$ is said to be nodal if its dual subdivision $S$ of $\Delta$ is such that

- all the points in $\partial \Delta \cap \mathbb{Z}^{2}$ are vertices of $S$;

- $S$ consists of triangles and parallelograms.

We define the weight of a nodal amoeba $A$ by the formula

$$
W(A)=\prod_{\substack{\Delta^{\prime} \in P(S) \\\left|V\left(\Delta^{\prime}\right)\right|=3}}\left|\Delta^{\prime}\right|,
$$

where $P(S)$ denotes the set of polygons of $S$, and $\left|\Delta^{\prime}\right|$ stands for the double Euclidean area of $\Delta^{\prime}$. 
Theorem 3. In the previous notation,

$$
\operatorname{deg} \Sigma_{\Delta}\left(n A_{1}\right)=\sum_{A} W(A),
$$

where the sum ranges over all nodal amoebas of rank $r$ passing through $r$ fixed generic points in $\mathbb{Q}^{2}$.

Remark 3.1. Our formula coincides with that given by Mikhalkin [18. Namely, the multiplicity of a lattice path defined in [18 is equal to the sum of the multiplicities of the nodal amoebas that correspond to the subdivisions of $\Delta$ arising from the given path in the course of the construction in 18 . Also, we note that the generality requirement for the position of $r$ points in $\mathbb{Q}^{2}$ will be specified in the proof, and it is easily checked that the configurations considered by Mikhalkin [19], i.e., generic points on a generic straight line, satisfy these generality conditions.

The proof comprises three main steps. First, we determine amoebas and tropicalizations of nodal curves in the count, in particular, that the amoebas are nodal of rank $r$ (Subsection 3.3). Then we refine tropicalizations in a suitable way (Subsections 3.5, 3.6). Finally, using the patchworking theorem, we show that the refined tropicalization gives rise to an explicit number of nodal curves passing through given points (Section 3.7).

3.2. Deformation of reducible surfaces and curves. We start with the following auxiliary statement.

Lemma 3.2. Suppose a complex threefold $Y$ is smooth at a point $z$, and $U \subset Y$ is a small ball centered at $z$. Assume that $\pi: U \rightarrow(\mathbb{C}, 0)$ is a flat family of reduced surfaces such that $U_{0}=\pi^{-1}(0)$ consists of two smooth components $U_{0}^{\prime}, U_{0}^{\prime \prime}$ that intersect transversally along a line $L \supset\{z\}$, and the $U_{t}=\pi^{-1}(t)$ are nonsingular for $t \neq 0$. Let $C_{0}^{\prime} \subset U_{0}^{\prime}$ and $C_{0}^{\prime \prime} \subset U_{0}^{\prime \prime}$ be reduced algebraic curves that cross $L$ only at $z$ and with the same multiplicity $m \geq 2$. Also, we assume that $U_{0}^{\prime}$ and $U_{0}^{\prime \prime}$ are regular neighborhoods of the (possibly singular) point $z$ of $C_{0}^{\prime}$ and $C_{0}^{\prime \prime}$, respectively. Let $\delta^{\prime}=\delta\left(C_{0}^{\prime}, z\right), \delta^{\prime \prime}=\delta\left(C_{0}^{\prime \prime}, z\right)$ be the $\delta$-invariants, and let $r^{\prime}, r^{\prime \prime}$ be the number of the local branches of $C_{0}^{\prime}$ and $C_{0}^{\prime \prime}$ at $z$, respectively. Then in any flat deformation $C_{t}, t \in(\mathbb{C}, 0)$, of $C_{0}=C_{0}^{\prime} \cup C_{0}^{\prime \prime}$ such that $C_{t} \subset U_{t}$, the total $\delta$-invariant of $C_{t}, t \neq 0$, in $U_{t}$ does not exceed

$$
\delta^{\prime}+\delta^{\prime \prime}+m-\max \left\{r^{\prime}, r^{\prime \prime}\right\} .
$$

Proof. Topologically, the curves $C_{0}^{\prime}$ and $C_{0}^{\prime \prime}$ (in $U$ ) are bouquets of $r^{\prime}$ and $r^{\prime \prime}$ disks, respectively. Observe that the circles of $C_{0}^{\prime} \cap \partial U$ and $C_{0}^{\prime \prime} \cap \partial U$ move slightly when $t$ changes, and they are not contractible in $U_{t}$ for $t \neq 0$. For instance, a circle of $C_{0}^{\prime} \cap \partial U$ is (positively) linked with the line $L$ in $U_{0}^{\prime}$, and, hence, it remains (positively) linked with the surface $U_{0}^{\prime \prime}$ in $U$; thus, it cannot be contracted in $U_{t}, t \neq 0$, which does not intersect $U_{0}^{\prime \prime}$. This means that the curve $C_{t} \subset U_{t}, t \neq 0$, is a union of several immersed surfaces with a total of $r^{\prime}+r^{\prime \prime}$ holes and at least $\max \left\{r^{\prime}, r^{\prime \prime}\right\}$ handles.

Now the claimed upper bound can be derived either from a local count of intersections and self-intersections of the components of $C_{t}$, or by a "global" argument. For the latter, we consider the following model situation, which is quite relevant to our consideration and is explored in more detail below in the proof of Theorem 3. Namely, assume that

- $p \gg m, p \in \mathbb{N}$;

- $\Delta \subset \mathbb{R}^{2}$ is the triangle with vertices $(0,0),(2 p, 0),(0,2 p)$;

- $\nu: \Delta \rightarrow \mathbb{R}$ is the function such that $\nu(\alpha, \beta)=0$ if $\alpha+\beta \leq p$, and $\nu(\alpha, \beta)=$ $\alpha+\beta-p$ if $p \leq \alpha+\beta$;

- $\widetilde{\Delta}=\left\{(\alpha, \beta, \gamma) \in \mathbb{R}^{3}:(\alpha, \beta) \in \Delta, \nu(\alpha, \beta) \leq \gamma \leq p+1\right\}$. 
Then $Y=\operatorname{Tor}(\widetilde{\Delta})$ is a nonsingular threefold, and $Y_{0}^{\prime}=\operatorname{Tor}\left(\Delta^{\prime}\right), Y_{0}^{\prime \prime}=\operatorname{Tor}\left(\Delta^{\prime \prime}\right)$ are surfaces isomorphic (respectively) to $\mathbb{P}^{2}$ and $\mathbb{P}^{2}$ with a blown-up point, where $\Delta^{\prime}=$ $\operatorname{conv}\{(0,0,0),(p, 0,0),(0, p, 0)\}, \Delta^{\prime \prime}=\operatorname{conv}\{(p, 0,0),(0, p, 0),(2 p, 0, p),(0,2 p, p)\}$ are the faces of $\operatorname{Graph}(\nu)$. Furthermore, $Y_{0}^{\prime}$ and $Y_{0}^{\prime \prime}$ intersect transversally along the line $L=$ $\operatorname{Tor}(\sigma), \sigma=[(p, 0,0),(0, p, 0)]$. A neighborhood $V$ of $Y_{0}=Y_{0}^{\prime} \cup Y_{0}^{\prime \prime}$ in $Y$ admits a fibration $V \rightarrow(\mathbb{C}, 0)$ such that the zero fiber $Y_{0}$ and the other fibers $Y_{t}$ are the closures of the images of the hyperplanes $\left\{x_{3}=t\right\} \subset\left(\mathbb{C}^{*}\right)^{3}$ under the standard embedding of $\left(\mathbb{C}^{*}\right)^{3}$ in $Y$ with the coordinate correspondence $(\alpha, \beta, \gamma) \leftrightarrow\left(x_{1}, x_{2}, x_{3}\right)$ of $\mathbb{R}^{3}$ and $\left(\mathbb{C}^{*}\right)^{3}$. Clearly, $Y_{t} \simeq \mathbb{P}^{2}, t \neq 0$. Assume that the curves $C_{0}^{\prime} \subset Y_{0}^{\prime}, C_{0}^{\prime \prime} \subset Y_{0}^{\prime \prime}$ are given by polynomials with Newton polygons $\Delta^{\prime}, \Delta^{\prime \prime}$, respectively, with a common truncation to $\sigma$, and such that they have a common point $z \in L$ as in the statement of the lemma, are nonsingular outside $z$, and intersect $L$ transversally outside $z$ (at common points). The flatness of a deformation $C_{t} \subset Y_{t}, t \in(\mathbb{C}, 0)$, of the curve $C_{0}=C_{0}^{\prime} \cup C_{0}^{\prime \prime}$ means that $C_{t}, t \neq 0$, tends to a curve of degree $2 p$ by the isomorphism $Y_{t} \simeq \mathbb{P}^{2}$. Denoting by $U$ a neighborhood of $C_{0}^{\prime} \cap C_{0}^{\prime \prime}$ in $Y$, for the Euler characteristic $\check{\chi}\left(C_{t}\right)$ of the normalization of $C_{t}$, we obtain the following bound:

$$
\begin{aligned}
\check{\chi}\left(C_{t}\right)= & \chi\left(C_{t} \backslash U\right)+\check{\chi}\left(C_{t} \cap U\right)=\chi\left(C_{0}^{\prime} \backslash U\right)+\chi\left(C_{0}^{\prime \prime} \backslash U\right)+\check{\chi}\left(C_{t} \cap U\right) \\
\leq & \left(-p^{2}+2 p+m-r^{\prime}+2 \delta^{\prime}\right)+\left(-3 p^{2}+4 p+m-r^{\prime \prime}+2 \delta^{\prime \prime}\right) \\
& \quad+\left(r^{\prime}+r^{\prime \prime}-2 \max \left\{r^{\prime}, r^{\prime \prime}\right\}\right) \\
& -4 p^{2}+6 p+2 m+2 \delta^{\prime}+2 \delta^{\prime \prime}-\max \left\{r^{\prime}, r^{\prime \prime}\right\} .
\end{aligned}
$$

Consequently, for the total $\delta$-invariant of $C_{t}$ we have

$$
\begin{aligned}
\delta\left(C_{t}\right) & =\frac{(2 p-1)(2 p-2)}{2}-g\left(C_{t}\right) \\
& =\frac{(2 p-1)(2 p-2)}{2}-1+\frac{\check{\chi}\left(C_{t}\right)}{2} \\
& \leq \delta^{\prime}+\delta^{\prime \prime}+m-\max \left\{r^{\prime}, r^{\prime \prime}\right\} .
\end{aligned}
$$

Example 3.3. In the notation of Lemma 3.2, if $C_{0}^{\prime}, C_{0}^{\prime \prime}$ are nonsingular at $z$, then $\delta_{1}=\delta_{2}=0, r_{1}=r_{2}=1$, and the number of nodes in a deformation does not exceed $m-1$; this number can be attained (see [3. Theorem 2.1]). We can produce the maximal number of nodes with the help of suitable deformation patterns (i.e., certain affine curves), as defined below in Subsection 3.5 .

Remark 3.4. In the notation of Lemma 3.2. suppose all the hypotheses are fulfilled, but assume that $C_{0}^{\prime}, C_{0}^{\prime \prime}$ are not necessarily reduced. Furthermore, let $C_{0}^{\prime}$ (respectively, $C_{0}^{\prime \prime}$ ) have $r^{\prime}$ (respectively, $r^{\prime \prime}$ ) reduced local branches at $z$ of multiplicities $\rho_{1}^{\prime}, \ldots, \rho_{r^{\prime}}^{\prime}$ (respectively, $\rho_{1}^{\prime \prime}, \ldots, \rho_{r^{\prime \prime}}^{\prime \prime}$ ). Then the argument used in the proof of Lemma 3.2 shows that if $C_{t}$ is reduced in $U$, then

$$
\check{\chi}\left(C_{t} \cap U\right) \leq-\min \left|m_{1}^{\prime}+\cdots+m_{r^{\prime}}^{\prime}-m_{1}^{\prime \prime}-\cdots-m_{r^{\prime \prime}}^{\prime \prime}\right|,
$$

where the integers $m_{1}^{\prime}, \ldots, m_{r^{\prime \prime}}^{\prime \prime}$ run over the range $1 \leq m_{1}^{\prime} \leq \rho_{1}^{\prime}, \ldots, 1 \leq m_{r^{\prime \prime}}^{\prime \prime} \leq \rho_{r^{\prime \prime}}^{\prime \prime}$.

3.3. Amoebas and tropicalizations of nodal curves passing through generic points. Let $\boldsymbol{x}_{1}, \ldots, \boldsymbol{x}_{r} \in \mathbb{Q}^{2}$ be generic distinct points, and let $\boldsymbol{p}_{1}, \ldots, \boldsymbol{p}_{r} \in\left(\mathbb{K}^{*}\right)^{2}$ be generic points satisfying $\operatorname{Val}\left(\boldsymbol{p}_{i}\right)=\boldsymbol{x}_{i}, i=1, \ldots, r$, and having only rational exponents of the parameter $t$.

The parameter change $t \mapsto t^{m}$ with a suitable natural $m$ makes all the exponents of $t$ in the coefficients of $f$ integral and the convex piecewise linear function $\nu_{f}: \Delta \rightarrow \mathbb{R}$ integral-valued at integral points. We keep these assumptions throughout the remaining part of the paper. 
Let $S_{f}: \Delta=\Delta_{1} \cup \cdots \cup \Delta_{N}$ be the subdivision determined by $\nu_{f}$, and let $\left(C_{1}, \ldots, C_{N}\right)$ be the tropicalization of the curve $C=\{f=0\} \in \Lambda_{K}(\Delta)$. The union of the divisors $\operatorname{Tor}(\sigma) \subset \operatorname{Tor}\left(\Delta_{k}\right)$, where $\sigma$ runs over all edges of $\Delta_{k}$, will be denoted by $\operatorname{Tor}\left(\partial \Delta_{k}\right)$, $k=1, \ldots, N$. For any $i=1, \ldots, N$, we denote by $C_{i j}, j=1, \ldots, m_{i}$, the distinct irreducible components of the curve $C_{i} \subset \operatorname{Tor}\left(\Delta_{i}\right)$ and by $r_{i j}, j=1, \ldots, m_{i}$, their multiplicities. Put $s_{i j}=\#\left(C_{i j} \cap \operatorname{Tor}\left(\partial \Delta_{i}\right)\right), j=1, \ldots, m_{i}$.

We intend to estimate $\check{\chi}\left(C^{(t)}\right)$ from above and from below and to compare these bounds.

Let $U$ be the union of small open balls $U_{z}$ in the threefold $Y$ (see the definition in Subsection 2.3) centered at all points $z \in \bigcup_{i}\left(C_{i} \cap \operatorname{Tor}\left(\partial \Delta_{i}\right)\right)$. If $z \in C_{i} \cap \operatorname{Tor}(\sigma)$, where $\sigma$ is an edge of $\Delta_{i}$ lying on $\partial \Delta$, then $\check{\chi}\left(C^{(t)} \cap U_{z}\right)$ does not exceed the number of the local branches of $C_{i}$ at the points of $C_{i} \cap \operatorname{Tor}(\sigma)$. If $z \in \operatorname{Tor}(\sigma) \cap C_{i} \cap C_{k}$, where $\sigma=\Delta_{i} \cap \Delta_{k}$ is a common edge, then $\check{\chi}\left(C^{(t)} \cap U_{z}\right) \leq 0$ by Remark 3.4. Consequently,

$$
\check{\chi}\left(C^{(t)} \cap U\right) \leq\left|\partial \Delta \cap \mathbb{Z}^{2}\right|,
$$

where equality occurs if and only if, for any edge $\sigma \subset \Delta_{i} \cap \partial \Delta$, the reduction of the curve $C_{i}$ is nonsingular along Tor $(\sigma)$ and meets $\operatorname{Tor}(\sigma)$ transversally.

For the upper bound of $\check{\chi}\left(C^{(t)}\right)$, we may assume that for any $i=1, \ldots, N$ and $1 \leq j<$ $j^{\prime} \leq m_{i}$ the components $C_{i j}$ and $C_{i j^{\prime}}$ do not join together in $Y \backslash U$ when $C^{(0)}$ deforms into $C^{(t)}$. Then the normalization of $C^{(t)} \backslash U$ is a union of connected components, each of them tending to some curve $C_{i j} \backslash U$. Furthermore, the components that tend to a certain $C_{i j} \backslash U$ can be naturally projected onto $C_{i j} \backslash U$, and this projection is an $r_{i j}$ sheeted covering (possibly ramified at a finite set). Hence,

$$
\begin{aligned}
\check{\chi}\left(C^{(t)} \backslash U\right) & \leq \sum_{i=1}^{N} \sum_{j=1}^{m_{i}} r_{i j} \check{\chi}\left(C_{i j} \backslash U\right)=\sum_{i=1}^{N} \sum_{j=1}^{m_{i}} r_{i j}\left(\check{\chi}\left(C_{i j}\right)-s_{i j}\right) \\
& \leq 2 \sum_{i=1}^{N} m_{i}-\sum_{i=1}^{N} \sum_{j=1}^{m_{i}} s_{i j},
\end{aligned}
$$

where equality occurs only if all $C_{i j}$ are rational, and $r_{i j}=1$ whenever $s_{i j}>2$. Next we observe that $s_{i j} \geq 2$ for any $C_{i j}$, and $s_{i j} \geq 3$ for at least one of the components $C_{i j}$ if $\Delta_{i}$ has an odd number of edges, or $\Delta_{i}$ has an even number of edges but not all pairs of opposite sides are parallel. Therefore (in the notation of Lemma 2.2),

$$
\check{\chi}\left(C^{(t)} \backslash U\right) \leq-N_{3}-\sum_{j \geq 2}\left(N_{2 j+1}+N_{2 j}-N_{2 j}^{\prime}\right),
$$

where equality occurs only if, for each triangle $\Delta_{i}, C_{i}$ is irreducible and satisfies $s_{i j}=3$. For each $\Delta_{i}$ with an odd number of edges exceeding 3 , or with an even number of edges, but such that not all pairs of opposite sides are parallel, exactly one component $C_{i j}$ satisfies $s_{i j}=3$ and the others satisfy $s_{i j}=2$; finally, we have $s_{i j}=2$ for all components $C_{i j}$ in the remaining polygons $\Delta_{i}$. Observe also that $s_{i j}=2$ means that $C_{i j}$ is defined by a binomial.

On the other hand,

$$
\begin{aligned}
\check{\chi}\left(C^{(t)}\right) & =2-2 g\left(C^{(t)}\right)=2-2\left(\left|\operatorname{Int}(\Delta) \cap \mathbb{Z}^{2}\right|-n\right) \\
& =2-2\left|\operatorname{Int}(\Delta) \cap \mathbb{Z}^{2}\right|+2\left(\left|\Delta \cap \mathbb{Z}^{2}\right|-1-r\right) \\
& =2\left|\partial \Delta \cap \mathbb{Z}^{2}\right|-2 r \geq 2\left|\partial \Delta \cap \mathbb{Z}^{2}\right|-2 \cdot \operatorname{rk}\left(S_{f}\right) \\
& =2\left|\partial \Delta \cap \mathbb{Z}^{2}\right|-2 \cdot \operatorname{rk}_{v}\left(S_{f}\right)-2 d\left(S_{f}\right)
\end{aligned}
$$


with equality only if $\operatorname{rk}\left(S_{f}\right)=\operatorname{rk}_{v}\left(S_{f}\right)+d\left(S_{f}\right)=r$. Next, by (2.2.2) we have

$$
\begin{aligned}
\check{\chi}\left(C^{(t)}\right) & \geq 2\left|\partial \Delta \cap \mathbb{Z}^{2}\right|-2\left|V\left(S_{f}\right)\right|+2+2 \sum_{i=1}^{N}\left(\left|V\left(\Delta_{i}\right)\right|-3\right)-2 d\left(S_{f}\right) \\
& =2\left|\partial \Delta \cap \mathbb{Z}^{2}\right|-2\left|V\left(S_{f}\right)\right|+2-2\left|V\left(S_{f}\right) \cap \partial \Delta\right|+4\left|E\left(S_{f}\right)\right|-6 N-2 d\left(S_{f}\right),
\end{aligned}
$$

where $E\left(S_{f}\right)$ denotes the set of edges of $S_{f}$. Since $\left|V\left(S_{f}\right)\right|-\left|E\left(S_{f}\right)\right|+N=1$, and $2\left|E\left(S_{f}\right)\right|=3 N_{3}+4 N_{4}+5 N_{5}+\cdots+\left|V\left(S_{f}\right) \cap \partial \Delta\right|$, finally we obtain

$$
\begin{aligned}
\check{\chi}\left(C^{(t)}\right) \geq & 2\left(\left|\partial \Delta \cap \mathbb{Z}^{2}\right|-\left|V\left(S_{f}\right) \cap \partial \Delta\right|\right) \\
& +\left|V\left(S_{f}\right) \cap \partial \Delta\right|-N_{3}+N_{5}+2 N_{6}+\cdots-2 d\left(S_{f}\right) .
\end{aligned}
$$

Combining this with (3.3.7) and (3.3.8) leads to the estimate

$$
\begin{aligned}
(\mid \partial \Delta & \left.\cap \mathbb{Z}^{2}|-| V\left(S_{f}\right) \cap \partial \Delta \mid\right)+\sum_{\left|V\left(\Delta_{i}\right)\right|=3}\left(m_{i}-1\right) \\
& +\sum_{m \geq 2}\left((2 m-3) N_{2 m}-N_{2 m}^{\prime}\right)+\sum_{m \geq 2}(2 m-2) N_{2 m+1} \leq 2 d\left(S_{f}\right),
\end{aligned}
$$

which, by Lemma 2.2, shows that each integral point on $\partial \Delta$ is a vertex of $S_{f}$ and all the nontriangular $\Delta_{i}$ are parallelograms.

Altogether, the equality conditions for the upper and lower bounds of $\check{\chi}\left(C^{(t)}\right)$ prove that the amoeba $A_{f}$ is nodal of rank $r$. Furthermore,

- for each triangle $\Delta_{i}$, the curve $C_{i}$ is rational and meets $\operatorname{Tor}\left(\partial \Delta_{i}\right)$ at exactly three points, where it is unibranch;

- for each parallelogram $\Delta_{i}$, the polynomial that defines $C_{i}$ has the form $x^{k} y^{l}\left(\alpha x^{a}+\beta y^{b}\right)^{p}\left(\gamma x^{c}+\delta y^{d}\right)^{q}$ with $(a, b)=(c, d)=1$ and $(a: b) \neq(c: d)$.

We shall describe these curves more precisely.

Lemma 3.5. For any lattice triangle $\Delta^{\prime} \subset \mathbb{R}^{2}$, there exists a polynomial with Newton polygon $\Delta^{\prime}$ with prescribed coefficients at the vertices of $\Delta^{\prime}$, and such that it defines a rational curve $C \subset \operatorname{Tor}\left(\Delta^{\prime}\right)$ meeting $\operatorname{Tor}\left(\partial \Delta^{\prime}\right)$ at exactly three points where it is unibranch. The curves defined by these polynomials are nodal and nonsingular at the intersection with $\operatorname{Tor}\left(\partial \Delta^{\prime}\right)$. Moreover, the number of such polynomials is finite and is equal to $\left|\Delta^{\prime}\right|$. An additional fixation of one or two intersection points of $C$ with $\operatorname{Tor}\left(\partial \Delta^{\prime}\right)$ divides the number of polynomials under consideration by the product of the lengths ${ }^{3}$ of the corresponding edges of $\Delta^{\prime}$.

Proof. By a suitable lattice-preserving transformation, we can turn $\Delta^{\prime}$ into a triangle with vertices $(p, 0),(q, 0),(0, m), 0 \leq p<q \leq m$. Assume that the curve $C$ crosses $\operatorname{Tor}(\partial \Delta)$ at points corresponding to the values 0,1 and $\infty$ of a uniformizing parameter $\theta$; then $C$ is given by $x=\alpha \theta^{m}, y=\beta \theta^{p}(\theta-1)^{q-p}$. If the restrictions of the defining polynomial on the edges $[(p, 0),(q, 0)]$ and $[(p, 0),(0, m)]$ are $x^{p}\left(x+\varepsilon_{1}\right)^{q-p}$ and $\left(y^{m / d}+\varepsilon_{2} x^{p / d}\right)^{d}$, respectively, where $d=\operatorname{gcd}(m, p), \varepsilon_{1}^{q-p}=\varepsilon_{2}^{d}=1$, then

$$
\alpha+\varepsilon_{1}=0, \quad \beta^{m / d}(-1)^{m(q-p) / d}+\varepsilon_{2} \alpha^{p / d}=0,
$$

which gives $m(q-p)=|\Delta|$ solutions for $(\alpha, \beta)$. Additional fixation of intersection points with $\operatorname{Tor}\left(\partial \Delta^{\prime}\right)$ means fixation of $\varepsilon_{1}$ or/and $\varepsilon_{2}$ and the respective reduction of the number of solutions. The prescribed coefficients of $x^{p}, x^{q}, y^{m}$ in the polynomial can be achieved by an appropriate coordinate change.

${ }^{3}$ We define the length $|\sigma|$ of a segment $\sigma$ with integral endpoints as $\left|\sigma \cap \mathbb{Z}^{2}\right|-1$. 
It remains to show that the curve $x=\theta^{m}, y=\theta^{p}(\theta-1)^{q-p}$ is nodal. Since $\dot{x}(\theta) \neq 0$ for $\theta \neq 0$, the curve has no local singular branches. Assuming that

$$
\theta^{m}=\theta_{1}^{m}=\theta_{2}^{m}, \quad \theta^{p}(\theta-1)^{q-p}=\theta_{1}^{p}\left(\theta_{1}-1\right)^{q-p}=\theta_{2}^{p}\left(\theta_{2}-1\right)^{q-p},
$$

we successively obtain

$$
\begin{gathered}
\theta_{1}=\theta \varepsilon_{1}, \quad \theta_{2}=\theta \varepsilon_{2}, \quad \varepsilon_{1}^{m}=\varepsilon_{2}^{m}=1, \quad \varepsilon_{1} \neq \varepsilon_{2}, \\
\theta-1=\varepsilon_{3}\left(\theta \varepsilon_{1}-1\right)=\varepsilon_{4}\left(\theta \varepsilon_{2}-1\right), \quad \varepsilon_{3}^{q-p}=\varepsilon_{1}^{p}, \quad \varepsilon_{4}^{q-p}=\varepsilon_{2}^{p}, \\
\theta=\frac{1-\eta_{1}^{p}}{1-\eta_{1}^{q}}=\frac{1-\eta_{2}^{p}}{1-\eta_{2}^{q}}
\end{gathered}
$$

where

$$
\begin{gathered}
\varepsilon_{1}=\eta_{1}^{q-p}, \quad \varepsilon_{3}=\eta_{1}^{p}, \quad \varepsilon_{2}=\eta_{2}^{q-p}, \quad \varepsilon_{4}=\eta_{2}^{p}, \\
\eta_{1}^{p} \neq 1, \quad \eta_{1}^{q-p} \neq 1, \quad \eta_{2}^{p} \neq 1, \quad \eta_{2}^{q-p} \neq 1 .
\end{gathered}
$$

Then, plugging $\eta_{1}=\cos \omega_{1}+\sqrt{-1} \sin \omega_{1}$ and $\eta_{2}=\cos \omega_{2}+\sqrt{-1} \sin \omega_{2}$ in (3.3.10), we get

$$
\frac{\cos \left(p \omega_{1} / 2\right) \cos \left(q \omega_{2} / 2\right)}{\cos \left(q \omega_{1} / 2\right) \cos \left(p \omega_{2} / 2\right)}=\cos \frac{(q-p)\left(\omega_{2}-\omega_{1}\right)}{2}+\sqrt{-1} \sin \frac{(q-p)\left(\omega_{2}-\omega_{1}\right)}{2},
$$

and finally,

$$
(q-p)\left(\omega_{2}-\omega_{1}\right) \in 2 \pi \cdot \mathbb{Z} \Longrightarrow \varepsilon_{1}=\eta_{1}^{q-p}=\eta_{2}^{q-p}=\varepsilon_{2}
$$

in contrast to the fact that $\varepsilon_{1} \neq \varepsilon_{2}$, and we are done.

Lemma 3.6. If $a, b, c, d$ are integers such that $(a, b)=(c, d)=1,(a: b) \neq(c: d)$, and $\alpha, \beta, \gamma, \delta$ are nonzero, then the curve $\left(\alpha x^{a}+\beta y^{b}\right)\left(\gamma x^{c}+\delta y^{d}\right)=0$ has $\left|\Delta^{\prime} \cap \mathbb{Z}^{2}\right|-3$ nodes as its only singularities in $\left(\mathbb{C}^{*}\right)^{2}$, where $\Delta^{\prime}$ is the lattice parallelogram built on the vectors $(a,-b),(c,-d)$.

Proof. Straightforward.

3.4. Irreducible curves and irreducible amoebas. One can talk of the nonArchimedean amoebas as the corner loci of all possible convex piecewise linear functions whose generic gradients are integral vectors (cf. [29]). In this sense, an amoeba is called reducible if it is the union of two proper sub-amoebas. The above description of the tropicalization of nodal curves has the following interesting consequence.

Lemma 3.7. In the notation of Subsection 3.3, the amoeba of an irreducible (respectively, reducible) r-nodal curve passing through $\boldsymbol{p}_{1}, \ldots, \boldsymbol{p}_{r}$ is irreducible (respectively, reducible).

Proof. The amoeba of a reducible curve is the union of the amoebas of the irreducible components, and thus, is reducible.

Let a nodal amoeba $A$ of rank $r$ be the union of distinct amoebas $A^{\prime}, A^{\prime \prime}$. The intersection points of $A^{\prime}$ and $A^{\prime \prime}$ are four-valent vertices of $A$, and they correspond to some parallelograms in the dual subdivision $\Delta=\Delta_{1} \cup \cdots \cup \Delta_{N}$. As was shown in Subsection 3.3, for any parallelogram $\Delta_{i}$, in the deformation $C^{(t)}, t \in(\mathbb{C}, 0)$, the distinct components of the curve $C_{i}$ do not join together. Consequently, the amoebas $A^{\prime}, A^{\prime \prime}$ are lifted up to separate algebraic curves. 
3.5. Refinement of tropicalization at an isolated singular point. In the notation and under the hypotheses of the preceding subsection, we shall introduce a refinement of the tropicalization for each point $z \in \operatorname{Tor}(\sigma)$, where $\sigma=\Delta_{k} \cap \Delta_{l}$ is a common edge, and the curves $C_{k}, C_{l}$ meet $\operatorname{Tor}(\sigma)$ at $z$ with multiplicity $m \geq 2$.

Let $\Delta_{k}, \Delta_{l}$ be triangles. Then $C_{k}$ and $C_{l}$ are nonsingular at $z$ and are tangent to $\operatorname{Tor}(\sigma)$ with multiplicity $m$. To cover the case of cuspidal curves, treated below in $\$ 4$ we consider a more general situation. Namely, assume that at $z$ the curve $C_{k}$ (respectively, $C_{l}$ ) has a semiquasihomogeneous singularity topologically equivalent to $y^{m_{1}}+x^{m}=0$, $m_{1} \leq m$ (respectively, $y^{m_{2}}+x^{m}=0, m_{2} \leq m$ ). We perform the following transformation of $f(x, y)$.

- Let $M_{\sigma}$ be an affine automorphism of $\mathbb{Z}^{2}$ that takes $\Delta$ to the right half-plane and takes $\sigma$ into a segment $\sigma^{\prime}$ on the horizontal coordinate axis. This corresponds to a monomial coordinate change $x=\left(x^{\prime}\right)^{a}\left(y^{\prime}\right)^{b}, y=\left(x^{\prime}\right)^{c}\left(y^{\prime}\right)^{d}$ in $f(x, y)$ and further multiplication by a monomial in $x^{\prime}, y^{\prime}$. The truncation of the new polynomial $f^{\prime}\left(x^{\prime}, y^{\prime}\right)$ on the edge $\sigma$ (i.e., the sum of the monomials of $f^{\prime}$ corresponding to the integral points in $\sigma$ ) is a polynomial in $x^{\prime}$ over $\mathbb{K}$. Its tropicalization is a complex polynomial in $x^{\prime}$, which is the common truncation of the tropicalizations $f_{k}^{\prime}$ and $f_{l}^{\prime}$ of $f^{\prime}$ on the polygons $M_{\sigma}\left(\Delta_{k}\right), M_{\sigma}\left(\Delta_{l}\right)$. The point $z$ corresponds to a root $\xi \neq 0$ of $P_{0}\left(x^{\prime}\right)$.

- Without loss of generality, assume that $\nu_{f}$ is zero along $\sigma$ (just multiply $f(x, y)$ by a suitable constant from $\left.\mathbb{K}^{*}\right)$. Then we perform the shift $x^{\prime}=x^{\prime \prime}+\xi, y^{\prime}=y^{\prime \prime}$, and put $f^{\prime \prime}\left(x^{\prime \prime}, y^{\prime \prime}\right)=f^{\prime}\left(x^{\prime}, y^{\prime}\right)$.

To understand the tropicalization of $f^{\prime \prime}$, we apply the above transformations to the polynomials

$$
P_{k}(x, y)=\sum_{(i, j) \in \Delta_{k}} c_{i j}^{0} t^{\lambda_{k}(i, j)} x^{i} y^{j}, \quad P_{l}(x, y)=\sum_{(i, j) \in \Delta_{l}} c_{i j}^{0} t^{\lambda_{l}(i, j)} x^{i} y^{j},
$$

where

$$
f_{k}(x, y)=\sum_{(i, j) \in \Delta_{k}} c_{i j}^{0} x^{i} y^{j}, \quad f_{l}(x, y)=\sum_{(i, j) \in \Delta_{l}} c_{i j}^{0} x^{i} y^{j}
$$

are the tropicalizations of $f$ on $\Delta_{k}, \Delta_{l}$, and $\lambda_{k}=\left.\nu_{f}\right|_{\Delta_{k}}$ and $\lambda_{l}=\left.\nu_{f}\right|_{\Delta_{l}}$ are linear functions. The Newton polygons $\Delta_{k}^{\prime \prime}$ and $\Delta_{l}^{\prime \prime}$ of the resulting polynomials ${ }^{4} P_{k}^{\prime \prime}$ and $P_{l}^{\prime \prime}$ contain segments $\left[(m, 0),\left(0, m_{1}\right)\right],\left[(m, 0),\left(0,-m_{2}\right)\right]$, respectively (see Figure 2).

Clearly, $\lambda_{k}^{\prime \prime}=\left.\nu_{f^{\prime \prime}}\right|_{\Delta_{k}^{\prime \prime}}$ and $\lambda_{l}^{\prime \prime}=\left.\nu_{f^{\prime \prime}}\right|_{\Delta_{l}^{\prime \prime}}$ are linear functions. Furthermore, $\nu_{f^{\prime \prime}}(i, j)>$ $\max \left\{\lambda_{k}^{\prime \prime}(i, j), \lambda_{l}^{\prime \prime}(i, j)\right\}$ for all points $(i, j)$ satisfying $0 \leq i<m, m_{1} i+m j<m m_{1}$, and $m_{2} i-m j<m m_{2}$ (i.e., inside the triangle $\Delta_{z}$ with vertices $\left.(m, 0),\left(0, m_{1}\right),\left(0,-m_{2}\right)\right)$. In particular, this means that the coefficients $c_{i j}^{\prime \prime}(t)$ of $\left(x^{\prime \prime}\right)^{i}\left(y^{\prime \prime}\right)^{j}$ in $f^{\prime \prime}$ satisfy $c_{m, 0}^{\prime \prime}(0) \neq 0$, $c_{i, 0}^{\prime \prime}(0)=0, i<m$. Hence, there is a unique $\tau(t) \in \mathbb{K}, \tau(0)=0$, such that the polynomial $\widetilde{f}(\widetilde{x}, \widetilde{y})=f^{\prime \prime}(\widetilde{x}+\tau(t), \widetilde{y})$ does not contain the monomial $\widetilde{x}^{m-1}$.

It is easily seen that $\left.\nu_{\tilde{f}}\right|_{\Delta_{k}^{\prime \prime}}=\lambda_{k}^{\prime \prime},\left.\nu_{\tilde{f}}\right|_{\Delta_{l}^{\prime \prime}}=\lambda_{l}^{\prime \prime}$, and $\nu_{\hat{f}}(i, j)>\max \left\{\lambda_{k}^{\prime \prime}(i, j), \lambda_{l}^{\prime \prime}(i, j)\right\}$ whenever $0 \leq i<m, m_{1} i+m j<m m_{1}$, and $m_{2} i-m j<m m_{2}$. It follows that the subdivision of the Newton polygon of $\widetilde{f}$ determined by the function $\nu_{\tilde{f}}$ contains a subdivision of the triangle $\Delta_{z}$; moreover, this subdivision has no vertices inside the segments $E_{k}^{z}=\left[(m, 0),\left(0, m_{1}\right)\right]$ and $E_{l}^{z}=\left[(m, 0),\left(0,-m_{2}\right)\right]$ and at the point $(m-1,0)$.

Finally, the fragment of the tropicalization of the polynomial $\widetilde{f}$ and of the curve $\widetilde{C}=$ $\{\tilde{f}=0\}$ restricted to the triangle $\Delta_{z}$ will be called the $z$-refinement of the tropicalization of $f$ and of $C$ and will be denoted by $\mathcal{T}_{z}(f), \mathcal{T}_{z}(C)$.

${ }^{4}$ From now on by "polynomial" we mean a Laurent polynomial. 


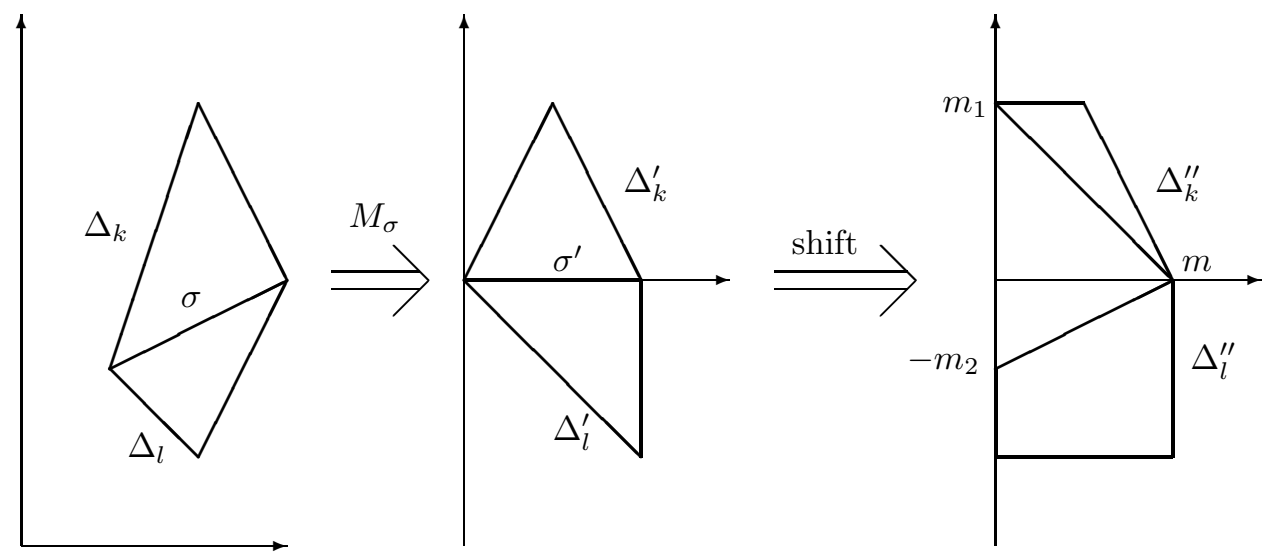

FIgURE 2. Refinement of the tropicalization, I

Remark 3.8. We note that the truncation $\varphi_{z}^{(k)}(x, y)$ (respectively, $\left.\varphi_{z}^{(l)}(x, y)\right)$ of the tropicalization of $\widetilde{f}$ on the segment $E_{k}^{z}$ (respectively, $E_{l}^{z}$ ) is uniquely determined by the polynomials $f_{k}, f_{l}$ and the point $z$. Any polynomial with Newton polygon $\Delta_{z}$ and such that its truncations to the edges $E_{k}^{z}, E_{l}^{z}$ are $\varphi_{z}^{(k)}, \varphi_{z}^{(l)}$ and the coefficient of $x^{m-1}$ vanishes will be called a deformation pattern compatible with $f_{k}, f_{l}$ and $z$.

In our situation, $C_{k}$ and $C_{l}$ are nonsingular at $z$, i.e., $m_{1}=m_{2}=1$, and the refinements of the tropicalization are described in the following statements.

Lemma 3.9. For a given integer $m \geq 2$ and fixed $a, b, c \in \mathbb{C}^{*}$, the set of polynomials $F(x, y)=a y^{2}+\operatorname{byg}(x)+c$ with $g(x)=x^{m}+\cdots, \operatorname{deg} g=m$, that define plane rational curves, consists of $m$ disjoint one-dimensional families. Each family has a unique representative with the zero coefficient of $x^{m-1}$ in $g(x)$, and the rest of the family can be obtained from that representative by the coordinate change $x \mapsto x+a, a \in \mathbb{C}$. Furthermore, all such rational curves have $m-1$ nodes in $\mathbb{C}^{2}$ as their only singularities.

Proof. Without loss of generality, suppose that $a=c=1, b=2$. Then the equations for singular points, $F(x, y)=F_{x}(x, y)=F_{y}(x, y)=0$, reduce to the system

$$
g(x)^{2}=1, \quad \frac{d g}{d x}(x)=0 .
$$

The solutions of this system are the $x$-coordinates of singular points, and their multiplicities in $g^{\prime}(x)$ are Milnor numbers. Hence, the total Milnor number does not exceed $m-1$. On the other hand, the total Milnor number is at least the $\delta$-invariant. Thus, by our assumptions, they coincide, which is only possible in the case of $m-1$ nodes corresponding to $m-1$ distinct solutions to (3.5.11). The latter condition on (3.5.11) is fulfilled if and only if $g(x)$ is (up to a shift $x \mapsto x+a$ ) the Chebyshev polynomial $\cos \left(m \cdot \arccos \left(2^{-(m-1) / m} x\right)\right)$ or one of the $m-1$ other polynomials of type $g(x \varepsilon), \varepsilon^{m}=1$, or $-g(x \varepsilon), \varepsilon^{m}=-1$.

Lemma 3.10. With the above notation and definitions, let $z \in \operatorname{Tor}(\sigma) \cap C_{k} \cap C_{l}$. If $C_{k}$ and $C_{l}$ are nonsingular at $z$, the intersection number of $C_{k}$ and $\operatorname{Tor}(\sigma)$ at $z$ is $\left(C_{k} \cdot \operatorname{Tor}(\sigma)\right)_{z}=$ $m \geq 2$, and $z$ bears singularities with the total $\delta$-invariant $m-1$, then $\mathcal{T}_{z}(f)$ consists of one polynomial having Newton polygon $\Delta_{z}$ and defining a rational curve with $m-1$ nodes in $\operatorname{Tor}\left(\Delta_{z}\right)$, and the singularities born of $z$ are $m-1$ nodes. 
Proof. We show that $\mathcal{T}_{z}(f)$ consists of the triangle $\Delta_{z}$ and, correspondingly, of one polynomial, which then is described in Lemma 3.9.

We use induction on $m$. Let $m=2$, and so $\mathcal{T}_{z}(f)$ contains more than one polynomial. Then $\Delta_{z}$ is subdivided into two triangles: $\operatorname{conv}\{(0,0),(0,1),(2,0)\}$ and $\operatorname{conv}\{(0,0)$, $(0,-1),(2,0)\}$. The curves defined by the polynomials with these Newton triangles are nonsingular and cross $\operatorname{Tor}([(0,0),(2,0)])$ transversally, because the coefficient of $x$ vanishes, but then no singular point appears in the deformation, by Lemma 3.2. Let $m \geq 3$, and let $\left(i_{1}, 0\right), \ldots,\left(i_{r}, 0\right) \in \operatorname{Int}\left(\Delta_{z}\right)$ be the vertices of the subdivision of $\Delta_{z}$ associated with $\mathcal{T}_{z}(f), r \geq 1,0 \leq i_{1}<\cdots<i_{r} \leq m-2$. Singular points may only appear from possible tangency points along $\operatorname{Tor}\left(\left[\left(i_{s}, 0\right),\left(i_{s+1}, 0\right)\right]\right), s=1, \ldots, r-1$, or along $\operatorname{Tor}\left(\left[\left(i_{r}, 0\right),(m, 0)\right]\right)$, or from a curve with Newton triangle conv $\left\{(0,1),(0,-1),\left(i_{1}, 0\right)\right\}$ if $i_{1}>0$. Since the curves with Newton triangles containing the edge $\left[\left(i_{r}, 0\right),(m, 0)\right]$ must cross $\operatorname{Tor}\left(\left[\left(i_{r}, 0\right),(m, 0)\right]\right)$ at least at two points, because of the condition that the coefficient of $\widetilde{x}^{m-1}$ vanishes, the total $\delta$-invariant of the singular points that may appear is at most

$$
\max \left\{0, i_{1}-1\right\}+\sum_{s=1}^{r-1}\left(i_{s+1}-i_{s}-1\right)+\left(m-i_{r}-2\right) \leq m-2,
$$

which gives a contradiction.

3.6. Refinement of the tropicalization along a nonisolated singularity. Assume that $z \in \operatorname{Tor}(\sigma), \sigma=\Delta_{k} \cap \Delta_{l}$ is a common edge, $\left(C_{k} \cdot \operatorname{Tor}(\sigma)\right)_{z}=\left(C_{l} \cdot \operatorname{Tor}(\sigma)\right)_{z}=$ $m \geq 2$, and at least one of the $\Delta_{k}, \Delta_{l}$ is a parallelogram. Then, after reenumeration, the pair $\Delta_{k}, \Delta_{l}$ extends up to a chain $\Delta_{1}, \ldots, \Delta_{p}, p \geq 3$, where $\Delta_{1}, \Delta_{p}$ are triangles, $\Delta_{2}, \ldots, \Delta_{p-1}$ are parallelograms, and $\sigma_{1}=\Delta_{1} \cap \Delta_{2}, \ldots, \sigma_{p-1}=\Delta_{p-1} \cap \Delta_{p}$ are common edges parallel to each other (see Figure 3(a)). We shall associate a refinement of the tropicalization with the union $Z$ of the multiple components of $C_{2}, \ldots, C_{p-1}$ that cross the lines $\operatorname{Tor}\left(\sigma_{1}\right), \ldots, \operatorname{Tor}\left(\sigma_{p-1}\right)$.

Multiplying $f(x, y)$ be a suitable constant belonging to $\mathbb{K}^{*}$, we can achieve the constancy of $\nu_{f}$ along the edges $\sigma_{1}, \ldots, \sigma_{p-1}$. Then we apply a transformation $M \in \operatorname{Aff}\left(\mathbb{Z}^{2}\right)$ that takes $\Delta$ to the right half-plane and makes $\sigma_{1}, \ldots, \sigma_{p-1}$ horizontal (see Figure 3(b)). The corresponding monomial coordinate change transforms $f(x, y)$ into a polynomial $f^{\prime}\left(x^{\prime}, y^{\prime}\right)$. For the latter polynomial, the truncations of the edges $\sigma_{1}, \ldots, \sigma_{p-1}$ of the tropicalizations of $\Delta_{1}, \ldots, \Delta_{p}$ contain a factor $(x-\xi)^{m}$ with some $\xi \in \mathbb{C}^{*}$. Then we introduce the polynomial $f^{\prime \prime}\left(x^{\prime \prime}, y^{\prime \prime}\right)=f^{\prime}\left(x^{\prime \prime}+\xi, y^{\prime \prime}\right)$ and consider its tropicalization. Again, for better understanding of $\mathcal{T}\left(f^{\prime \prime}\right)$, we apply the above coordinate changes to the polynomials

$$
P_{k}(x, y)=\sum_{(i, j) \in \Delta_{k}} c^{0} t^{\lambda_{k}(i, j)} x^{i} y^{j}, \quad k=1, \ldots, p
$$

where

$$
f_{k}(x, y)=\sum_{(i, j) \in \Delta_{k}} c^{0} x^{i} y^{j}, \quad \lambda_{k}=\left.\nu_{f}\right|_{\Delta_{k}}, k=1, \ldots, p .
$$

The Newton polygons $\Delta_{k}^{\prime \prime}$ of the resulting polynomials $P_{k}^{\prime \prime}\left(x^{\prime \prime}, y^{\prime \prime}\right), i=1, \ldots, p$, look as shown in Figure 3(c) and bound a trapezoid $\theta$ with vertices $(0, a-1),(0, b+1),(m, a)$, and $(m, b)$. Then, in particular, the functions $\left.\nu_{f^{\prime \prime}}\right|_{\Delta_{k}^{\prime \prime}}=\lambda_{k}^{\prime \prime}, k=1, \ldots, p$, are linear, and

$$
\nu_{f^{\prime \prime}}(i, j)>\max _{1 \leq k \leq p} \lambda_{k}^{\prime \prime}(i, j), \quad 0 \leq i<m, a \leq j \leq b .
$$

Now we consider subdivisions of $\theta$ into parallelograms and one triangle with edges parallel to the edges of $\theta$ (see, e.g., Figure 3(d), (e)). Exactly one of them can be induced by a convex piecewise linear function, defined as $\nu_{f^{\prime \prime}}$ on $\Delta_{1}^{\prime \prime} \cup \cdots \cup \Delta_{p}^{\prime \prime}$ and extended to $\theta$. Here we suppose that the function $\nu_{f}$ is generic among the convex piecewise linear 
functions determining the same subdivision $S_{f}$ of $\Delta$ (this means that it had generic rational slopes before we multiplied it by a large natural number), and then, necessarily, its graph has a break along the edges of the triangle. Let $(m, d)$ be a vertex of the triangle on the chosen subdivision of $\theta$. We perform one more shift. Namely, inequality (3.6.12) and the fact that the linear functions $\lambda_{k}^{\prime \prime}, k=1, \ldots, p$, are constant in the horizontal direction, show that there exists a unique $\tau(t) \in \mathbb{K}, \tau(0)=0$, such that the polynomial $\widetilde{f}(\widetilde{x}, \widetilde{y})=f^{\prime \prime}(\widetilde{x}+\tau(t), \widetilde{y})$ has no monomial $\widetilde{x}^{m-1} \widetilde{y}^{d}$ (next to the vertex of the triangle).

We claim that the function $\nu_{\tilde{f}}$ determines a subdivision of $\theta$ into one triangle and $p-1$ parallelograms as described above. Furthermore, the tropicalizations of $\tilde{f}$ on the parallelograms inside $\theta$ are products of binomials, and the tropicalization of $\widetilde{f}$ on the triangle inside $\theta$ (which we denote $\Delta_{Z}$ ) is $\widetilde{y}^{d} P(\widetilde{x}, \widetilde{y})$, where $P$ is a polynomial occurring in Lemma 3.5, with the vanishing coefficient of $\widetilde{x}^{m-1} \widetilde{y}$.

Indeed, deformation of the tropicalization of the curve $\widetilde{C}=\{\widetilde{f}=0\}$ on the polygons $\Delta^{\prime} \subset \theta$ and $\Delta^{\prime} \in P\left(S_{\widetilde{f}}\right)$ describes a deformation of the tropicalization $C^{(0)}$ of the original curve $C=\{f=0\}$ in a neighborhood of $Z$. The argument in Subsection 3.3 implies that the truncations of the tropicalization of $\widetilde{f}$ on the vertical edges of $\Delta_{2}^{\prime \prime}, \ldots, \Delta_{p-1}^{\prime \prime}$, lying on $\partial \theta$ (see Figure $3(\mathrm{c})$ ), determine irreducible components of the restriction of $\mathcal{T}(\widetilde{C})$ to the polygons subdividing $\theta$. In particular, each vertical edge of $\Delta_{2}^{\prime \prime}, \ldots, \Delta_{p-1}^{\prime \prime}$ lying on $\partial \theta$ is joined to a segment lying on the vertical coordinate axis by a sequence of parallelograms. Furthermore, the argument in Subsection 3.3 shows that all other components of $\mathcal{T}(\widetilde{C})$ in $\operatorname{Tor}\left(\Delta^{\prime}\right), \Delta^{\prime} \subset \theta$, must be rational, and each of them crosses $\bigcup_{\sigma} \operatorname{Tor}(\sigma)$ at two points; here $\sigma$ runs over all nonvertical edges of the subdivision $S_{\widetilde{f}}$ in $\theta$. This leaves for $\left.\mathcal{T}(\widetilde{C})\right|_{\theta}$ only the possibility claimed above. For instance, the subdivision in question cannot be as shown by dashes in Figure 3(f), because the tropicalization on $\widetilde{f}$ of the horizontal dashed segment $\sigma$, lying at the level $d$, cannot be a power of a binomial (observe that the monomial $\widetilde{x}^{m-1} \widetilde{y}^{d}$ is absent). Thus, the component of $\mathcal{T}(\widetilde{C})$ corresponding to $\sigma$ is irreducible and crosses Tor $(\sigma)$ at least at two points.

Remark 3.11. The truncations $\varphi_{Z}^{(1)}$ and $\varphi_{Z}^{(p)}$ of the tropicalization of $\tilde{f}$ on the nonvertical edges of the triangle $\Delta_{Z}$ are uniquely determined by the polynomials $f_{1}$ and $f_{p}$, respectively. Any polynomial with Newton triangle conv $\{(0,0),(m, 1),(0,2)\}\left(\Delta_{Z}\right.$ shifted down) whose truncations to the nonvertical edges coincide with $\varphi_{Z}^{(1)}, \varphi_{Z}^{(p)}$ (up to multiplication by a suitable monomial), and the coefficient of $x^{m-1} y$ vanishes, will be called a deformation pattern for the set $Z$.

3.7. Restoring a nodal curve out of tropical data. We denote by $\mathcal{Q}_{\Delta}\left(n A_{1}\right)$ the set of quadruples $(A, S, F, R)$, where

- $A \in \mathcal{A}(\Delta)$ is a nodal amoeba of rank $r, S: \Delta=\Delta_{1}, \ldots, \Delta_{N}$ is a subdivision of $\Delta$ dual to $A$, and $F, R$ are collections of polynomials in $\mathbb{C}[x, y]$, which together are defined up to multiplication by the same nonzero (complex) constant;

- $F=\left(f_{1}, \ldots, f_{N}\right)$, where $f_{i}$ is a polynomial with Newton polygon $\Delta_{i}, i=$ $1, \ldots, N$, and such that if $\Delta_{i}$ is a triangle, then $f_{i}$ defines a rational curve in $\operatorname{Tor}\left(\Delta_{i}\right)$ as described in Lemma 3.5, if $\Delta_{i}$ is a parallelogram, then $f_{i}$ defines a curve in $\operatorname{Tor}\left(\Delta_{i}\right)$ as described in Lemma 3.6. and for any common edge $\sigma=\Delta_{i} \cap \Delta_{j}$ the truncations $f_{i}^{\sigma}$ and $f_{j}^{\sigma}$ coincide;

- $R$ is a collection of deformation patterns compatible with $F$ as described in Remarks 3.8 and 3.11 .

We are given points $\boldsymbol{x}_{1}, \ldots, \boldsymbol{x}_{r} \in \mathbb{Q}^{2}$ and $\boldsymbol{p}_{1}, \ldots, \boldsymbol{p}_{r} \in\left(\mathbb{K}^{*}\right)^{2}$ such that $\operatorname{Val}\left(\boldsymbol{p}_{i}\right)=\boldsymbol{x}_{i}$, $i=1, \ldots, r$, and we intend to find out 


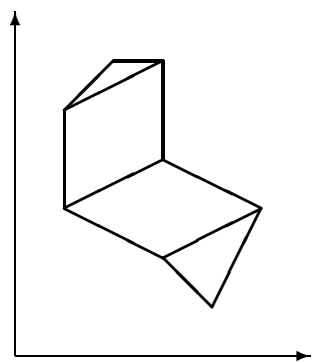

(a)

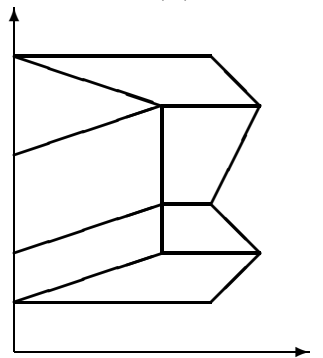

(d)

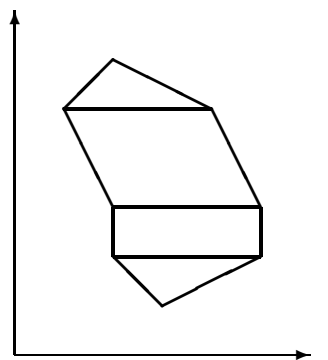

(b)

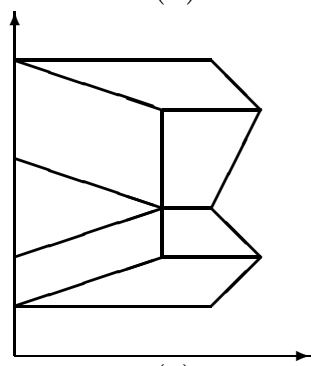

(e)

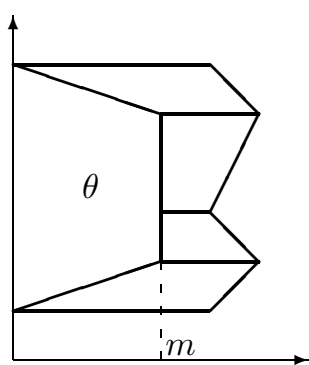

(c)

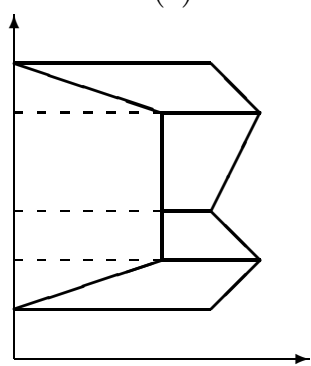

(f)

FiguRE 3. Refinement of the tropicalization, II

- how many elements $(A, S, F, R) \in \mathcal{Q}_{\Delta}\left(n A_{1}\right)$ correspond to a nodal amoeba $A \in$ $\mathcal{A}(\Delta)$ of rank $r$ passing through $\boldsymbol{x}_{1}, \ldots, \boldsymbol{x}_{r}$, and

- how many polynomials $f \in \mathbb{K}[x, y]$ (determined up to multiplication by a nonzero $\mathbb{K}$-constant) with Newton polygon $\Delta$ and defining curves $C \in \Sigma_{\Delta}\left(n A_{1}\right)$ that pass through $\boldsymbol{p}_{1}, \ldots, \boldsymbol{p}_{r}$ arise from a tropicalization $(A, S, F, R) \in \mathcal{Q}_{\Delta}\left(n A_{1}\right)$.

Step 1. Let $A \in \mathcal{A}(\Delta)$ be a nodal amoeba of rank $r$ passing through the given points $\boldsymbol{x}_{1}, \ldots, \boldsymbol{x}_{r} \in \mathbb{Q}^{2}$.

First, observe that $A$ uniquely determines a dual subdivision $S$ of $\Delta$. Indeed, the unbounded components of $\mathbb{R}^{2} \backslash A$ are in a natural one-to-one correspondence with $\partial \Delta \cap \mathbb{Z}^{2}$. The bounded edges of $A$ in the boundary of the above components determine germs of the edges of $S$ starting at $\partial \Delta \cap \mathbb{Z}^{2}$. There is a pair of nonparallel neighboring germs that start at distinct points of $\partial \Delta \cap \mathbb{Z}^{2}$, and their extension uniquely determines a triangle or a parallelogram in $P(S)$. Then we remove this polygon from $\Delta$ and continue the process.

Second, $A$ determines (uniquely up to a constant shift) a convex piecewise linear function $\nu: \Delta \rightarrow \mathbb{R}$ whose graph projects onto the subdivision $S$. More precisely, the points $\boldsymbol{x}_{1}, \ldots, \boldsymbol{x}_{r}$ lie on $r$ distinct edges of $A$ corresponding to some $r$ edges of $S$. If $\sigma_{i} \in E(S)$ corresponds to a point $\boldsymbol{x}_{i}$, and $\omega_{i}^{\prime}, \omega_{i}^{\prime \prime}$ are the endpoints of $\sigma_{i}, 1 \leq i \leq r$, then we have the following linear conditions on $\nu\left(\omega_{i}^{\prime}\right)$ and $\nu\left(\omega_{i}^{\prime \prime}\right)$ :

$$
\nu\left(\omega_{i}^{\prime}\right)-\nu\left(\omega_{i}^{\prime \prime}\right)=\left(\omega_{i}^{\prime \prime}-\omega_{i}^{\prime}\right) \boldsymbol{x}_{i}, \quad i=1, \ldots, r .
$$

Since $\boldsymbol{x}_{1}, \ldots, \boldsymbol{x}_{r}$ are generic, system (3.7.13) is independent. Furthermore, the parallelograms $\Delta_{j} \in P(S), j=1, \ldots, N_{4}$, corresponding to the 4 -valent vertices of $A$, impose the following linear conditions on the values of $\nu$ at the vertices $\omega_{j}^{(1)}, \omega_{j}^{(2)}, \omega_{j}^{(3)}, \omega_{j}^{(4)}$ of $\Delta_{j}$ (listed, say, clockwise):

$$
\nu_{f}\left(\omega_{j}^{(1)}\right)+\nu_{f}\left(\omega_{j}^{(3)}\right)=\nu_{f}\left(\omega_{j}^{(2)}\right)+\nu_{f}\left(\omega_{j}^{(4)}\right), \quad j=1, \ldots, N_{4} .
$$


Lemma 2.2 shows that the united system (3.7.13), (3.7.14) is independent, and, since it contains $|V(S)|-1$ equations, it determines the values of $\nu$ at the vertices of $S$ uniquely up to a constant shift.

Step 2. We are looking for polynomials of the form

$$
f(x, y)=\sum_{(i, j) \in \Delta} \widetilde{c}_{i j}(t) t^{\nu(i, j)} x^{i} y^{j}, \quad \widetilde{c}_{i j}(0)=c_{i j}, \quad(i, j) \in \Delta,
$$

where

$$
f_{k}(x, y)=\sum_{(i, j) \in \Delta_{i}} c_{i j} x^{i} y^{j}, \quad k=1, \ldots, N
$$

We claim that the condition

$$
f\left(\boldsymbol{p}_{1}\right)=\cdots=f\left(\boldsymbol{p}_{r}\right)=0
$$

determines the coefficients of $f_{1}, \ldots, f_{N}$ at the vertices of $S$, together with the truncations of $f_{1}, \ldots, f_{N}$ on the edges $\sigma_{1}, \ldots, \sigma_{r}$ corresponding to $\boldsymbol{x}_{1}, \ldots, \boldsymbol{x}_{r}$, uniquely up to multiplication by the same nonzero constant.

Indeed, let

$$
\begin{gathered}
\boldsymbol{x}_{i}=\left(-\alpha_{i},-\beta_{i}\right), \quad \boldsymbol{p}_{i}=(\xi, \eta), \quad \xi=\xi_{i}^{0} t_{i}^{\alpha}+\text { h.o.t. } \\
\eta=\eta_{i}^{0} t_{i}^{\beta}+\text { h.o.t., } \quad \xi_{i}^{0}, \eta_{i}^{0} \in \mathbb{C}^{*}
\end{gathered}
$$

and let $\omega_{i}^{\prime}=\left(i_{1}, j_{1}\right)$ and $\omega_{i}^{\prime \prime}=\left(i_{2}, j_{2}\right)$ be the endpoints of the edge $\sigma_{i}$. The conditions $f\left(\boldsymbol{p}_{i}\right)=0, i=1, \ldots, r$, take the form of the following equations:

$$
f\left(\boldsymbol{p}_{i}\right)=t^{\nu_{f}\left(i_{1}, j_{1}\right)+i_{1} \alpha_{i}+j_{1} \beta_{i}}\left(g_{i}\left(\xi_{i}^{0}, \eta_{i}^{0}\right)+O(t)\right)=0 \Longrightarrow g_{i}\left(\xi_{i}^{0}, \eta_{i}^{0}\right)=0,
$$

where a quasihomogeneous polynomial $g_{i}(x, y)=c_{\omega_{i}^{\prime}} x^{i_{1}} y^{j_{1}}+\cdots+c_{\omega_{i}^{\prime \prime}} x^{i_{2}} y^{j_{2}}$ is the tropicalization of $f^{\sigma_{i}}$. Since $g_{i}$ is the product of a monomial and a power of an irreducible binomial, (3.7.17) determines it uniquely up to a constant factor. On the other hand, for the coefficients $b_{1}, b_{2}, b_{3}, b_{4}$ of the polynomial $f_{j}$ having Newton parallelogram $\Delta_{j}$ with respective clockwise ordered vertices $\omega_{j}^{(1)}, \omega_{j}^{(2)}, \omega_{j}^{(3)}, \omega_{j}^{(4)}$, we have $b_{1} b_{3}=b_{2} b_{4}$. We see that the conditions imposed on the coefficients of $f_{1}, \ldots, f_{N}$ at $V(S)$ constitute a multiplicative form of a system such as (3.7.13), (3.7.14), whence the claim follows.

Next we compute in how many ways $f_{1}, \ldots, f_{N}$ can be restored if we fix the coefficients of the polynomials $f_{1}, \ldots, f_{N}$ at $V(S)$ and their truncations to the edges $\sigma_{1}, \ldots, \sigma_{r}$. To formulate the answer, we split $E(S)$ into disjoint subsets, assuming that two parallel edges of a parallelogram always belong to the same subset, and extending this relation by transitivity. ${ }^{5}$ Using Lemmas 3.5] and 3.6, it is not difficult to show (see, e.g., 27.) that there are $W(A) \prod_{\sigma}|\sigma|^{-1} \prod_{i=1}^{r}\left|\sigma_{i}\right|^{-1}$ collections of polynomials $f_{1}, \ldots, f_{N}$ compatible with the given data, where in the first product $\sigma$ ranges over a set of representatives of the subsets of $E(S)$ introduced above. Omitting the details, we explain this as follows. Fixing only coefficients at $V(S)$, we can obtain $W(A) \prod_{\sigma}|\sigma|^{-1}$ suitable combinations of $f_{1}, \ldots, f_{N}$ when recovering $f_{1}, \ldots, f_{N}$ inductively along the order used in the proof of Lemma 2.2. Then we observe that fixation of the truncation on each edge $\sigma_{i}, 1 \leq i \leq r$, divides the number of possibilities for $f_{1}, \ldots, f_{N}$ by $\left|\sigma_{i}\right|$.

Finally, by Lemma 3.9, we can find $W(A) \prod_{i=1}^{r}\left|\sigma_{i}\right|^{-1}$ elements $(A, S, F, R) \in \mathcal{Q}_{\Delta}\left(n A_{1}\right)$ compatible with the given nodal amoeba $A$ and the points $\boldsymbol{x}_{1}, \ldots, \boldsymbol{x}_{r} \in \mathbb{Q}^{2}, \boldsymbol{p}_{1}, \ldots, \boldsymbol{p}_{r} \in$ $\left(\mathbb{K}^{*}\right)^{2}$.

\footnotetext{
${ }^{5}$ In fact, a subset of $E(S)$ consists of edges parallel to each other and contained in parallelograms that form a chain in the sense of Subsection 3.6
} 
Step 3. We complete the proof of Theorem 3 with the following statement, which will be proved after the main patchworking Theorem 5 in Subsection 5.4.

Lemma 3.12. In the above notation, for given points $\boldsymbol{x}_{1}, \ldots, \boldsymbol{x}_{r} \in \mathbb{Q}^{2}, \boldsymbol{p}_{1}, \ldots, \boldsymbol{p}_{r} \in$ $\left(\mathbb{K}^{*}\right)^{2}$ and a compatible $(A, S, F, R) \in \mathcal{Q}_{\Delta}\left(n A_{1}\right)$, the polynomials $f \in \mathbb{K}[x, y]$ with Newton polygon $\Delta$ that tropicalize into $(A, S, F, R)$ define exactly $\prod_{i=1}^{r}\left|\sigma_{i}\right|$ curves in $\Lambda_{\mathbb{K}}(\Delta)$ having $n$ nodes and passing through $\boldsymbol{p}_{1}, \ldots, \boldsymbol{p}_{r}$.

\section{$\S 4$. Counting Curves with one CusP}

4.1. Formulation of the result. Let $\Delta$ be a nondegenerate convex lattice polygon having at least one interior integral point. We consider the variety $\Sigma_{\Delta}\left(A_{2}\right)$ of curves $C \in \Lambda_{\mathbb{K}}(\Delta)$ having an ordinary cusp as their only singularity, and we are going to express its degree as the number of certain non-Archimedean amoebas.

An amoeba $A \in \mathcal{A}(\Delta)$ is said to be 1-cuspidal if its dual subdivision $S$ of $\Delta$ satisfies one of the following conditions:

(i) $S$ contains a quadrangle Aff( $\left(\mathbb{Z}^{2}\right)$-equivalent to that shown in Figure 4 (a), and the rest of $S$ consists of triangles of area $1 / 2$;

(ii) $S$ contains a triangle $\mathrm{Aff}\left(\mathbb{Z}^{2}\right)$-equivalent to that shown in Figure 4 (b), and the rest of $S$ consists of triangles of area $1 / 2$;

(iii) $S$ contains an edge of length 2 common for a triangle $\operatorname{Aff}\left(\mathbb{Z}^{2}\right)$-equivalent to that shown in Figure 4 (c) and a triangle of area 1, and the rest of $S$ consists of triangles of area $1 / 2$;

(iv) $S$ contains an edge of length 2 common for a quadrangle Aff $\left(\mathbb{Z}^{2}\right)$-equivalent to that shown in Figure 4(d) and a triangle of area 1, and the rest of $S$ consists of triangles of area $1 / 2$;

(v) $S$ contains an edge of length 3 common for two triangles of area $3 / 2$, and the rest of $S$ consists of triangles of area $1 / 2$.

Observe that a 1-cuspidal amoeba has rank $r=\left|\mathbb{Z}^{2} \cap \Delta\right|-3$ and determines the dual subdivision uniquely.

Let $\boldsymbol{x}_{1}, \ldots, \boldsymbol{x}_{r}$ be generic points in $\mathbb{Q}^{2}$, and let $A$ be a 1 -cuspidal amoeba passing through these points. We shall introduce the weight $W\left(A, \boldsymbol{x}_{1}, \ldots, \boldsymbol{x}_{r}\right)$.

Assume that $A$ has only 3 -valent vertices, i.e., $S$ contains only triangles. Then we put $W\left(A, \boldsymbol{p}_{1}, \ldots, \boldsymbol{p}_{r}\right)$ equal to 5,6 , or 6 in accordance with cases (ii), (iii), or (v) in the definition of 1-cuspidal amoebas.

Assume that $A$ contains a 4 -valent vertex. The vertices of $S$ and the $r$ edges of $S$ dual to the edges of $A$ that contain the fixed points form a graph $\Gamma$. Observe that $\Gamma$ has no cycles, since otherwise, as shown in Step 2 in Subsection 3.7 we would have a dependent sequence of relations of type (3.7.13), which is impossible because of the generic choice of $\boldsymbol{p}_{1}, \ldots, \boldsymbol{p}_{r}$. Thus, the relation $|V(S)|=\left|\Delta \cap \mathbb{Z}^{2}\right|-1=r+2$ implies that $\Gamma$ consists of two disjoint trees (a tree may consist of one point). Furthermore, the vertices of the quadrangle $\Delta^{\prime}$ in $S$ dual to the 4-valent vertex of $A$ cannot all belong to one component of $\Gamma$, and in the case of $\Delta^{\prime}$ shown in Figure 4(d) it cannot happen that the two upper vertices belong to one component of $\Gamma$ and the lower vertices belong to the other. We take the vectors joining the vertices of $\Delta^{\prime}$ that belong to the same component of $\Gamma$, take one vector $v$ joining two vertices from distinct components of $\Gamma$, and denote by $w\left(A, \boldsymbol{x}_{1}, \ldots, \boldsymbol{x}_{r}\right)$ the minimal positive coefficient of $v$ in the possible integral linear combinations of all the vectors taken. Now we put $W\left(A, \boldsymbol{x}_{1}, \ldots, \boldsymbol{x}_{r}\right)$ equal to $w\left(A, \boldsymbol{x}_{1}, \ldots, \boldsymbol{x}_{r}\right)$ or $3 w\left(A, \boldsymbol{x}_{1}, \ldots, \boldsymbol{x}_{r}\right)$ in cases (i) or (iv), respectively.

Theorem 4. In the above notation,

$$
\operatorname{deg} \Sigma_{\Delta}\left(A_{2}\right)=\sum W\left(A, \boldsymbol{x}_{1}, \ldots, \boldsymbol{x}_{n}\right),
$$




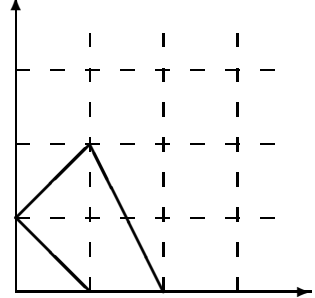

(a)

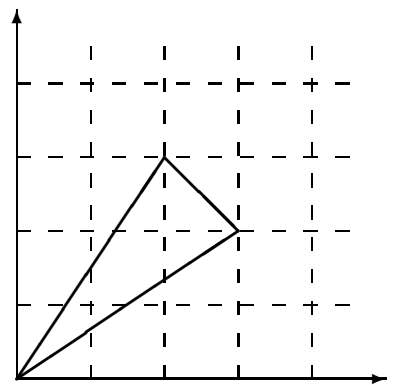

(b)

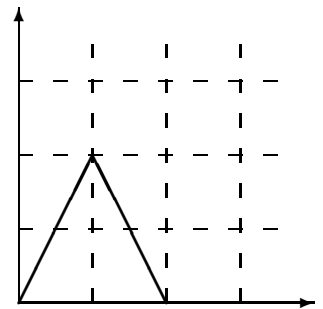

(c)

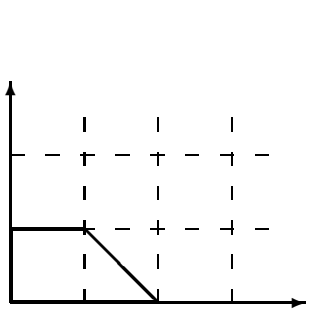

(d)

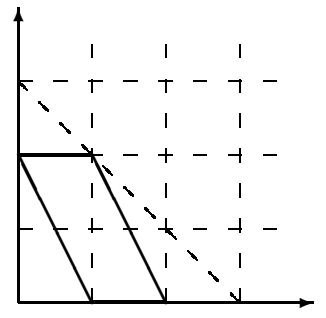

(e)

FigurE 4. Newton polygons of auxiliary curves with nodes and cusps

where $\boldsymbol{x}_{1}, \ldots, \boldsymbol{x}_{r} \in \mathbb{Q}^{2}$ is a collection of generic distinct points, and $A$ ranges over all 1-cuspidal amoebas in $\mathcal{A}(\Delta)$ passing through $\boldsymbol{x}_{1}, \ldots, \boldsymbol{x}_{r}$.

4.2. Auxiliary curves with nodes and cusps. We start by describing the nodal complex curves that will be used in the proof of Theorem 4

Lemma 4.1. Up to the action of the group $\mathrm{Aff}\left(\mathbb{Z}^{2}\right)$ of affine automorphisms of $\mathbb{Z}^{2}$,

- the polygons in Figure $4(\mathrm{a})$, (e) are the only lattice quadrangles with one interior integral point and all edges of length 1;

- the polygon in Figure $4(\mathrm{~b})$ is the only lattice triangle with two interior integral points and all edges of length 1 ;

- the polygon in Figure 4(c) is the only lattice triangle with one interior integral point, one edge of length 2 , and the other of length 1 ;

- the polygon in Figure $4(\mathrm{~d})$ is the only lattice quadrangle without interior integral points, with one edge of length 2, and the other of length 1.

There is no lattice pentagon whose vertices are its only integral points.

This is an elementary geometric fact, and we omit the proof.

Lemma 4.2. Let $\Delta_{i}, i=1, \ldots, 5$, denote the polygons shown in Figure 4(a)-(e), respectively.

(i) A curve in $\operatorname{Tor}\left(\Delta_{i}\right)$ defined by a polynomial with Newton polygon $\Delta_{i}$ cannot have a singularity more complicated than an ordinary cusp if $i=1,2$, and has at most one node if $i=3,4$.

(ii) For given coefficients at the vertices of $\Delta_{2}$, there exist exactly five polynomials with Newton triangle $\Delta_{2}$ that define a curve with a cusp. Furthermore, such curves have no other singular points. 
(iii) For given coefficients at the vertices of $\Delta_{3}$, there exist exactly two polynomials with Newton triangle $\Delta_{3}$ that define a curve with a node on $\operatorname{Tor}([(0,0),(2,0)])$.

(iv) A curve in $\operatorname{Tor}\left(\Delta_{1}\right)$ defined by a polynomial with Newton polygon $\Delta_{1}$ and coefficients $c_{10}, c_{01}, c_{12}, c_{20} \in \mathbb{C}^{*}$ at the vertices of $\Delta_{1}$ has a cusp if and only if

$$
c_{10}^{3} c_{1,2}=c_{01}^{2} c_{20}^{2} .
$$

Moreover, for fixed $c_{10}, c_{01}, c_{12}, c_{20}$ such a polynomial is unique, and the corresponding curve has a cusp as its only singularity.

(v) A curve in $\operatorname{Tor}\left(\Delta_{4}\right)$ defined by a polynomial with Newton polygon $\Delta_{4}$ and coefficients $c_{00}, c_{01}, c_{1,1}, c_{2,0} \in \mathbb{C}^{*}$ at the vertices of $\Delta_{4}$ has a node on $\operatorname{Tor}([(0,0),(2,0)])$ if and only if

$$
c_{00} c_{1,1}^{2}=c_{01}^{2} c_{20} .
$$

Moreover, for fixed $c_{00}, c_{01}, c_{1,1}, c_{2,0}$ such a polynomial is unique, and the corresponding curve has a node as its only singularity.

(vi) A curve in $\operatorname{Tor}\left(\Delta_{5}\right)$ defined by a polynomial with Newton polygon $\Delta_{5}$ cannot have cusps.

Proof. These statements are verified by direct computation, and we only explain (vi). Indeed, a polynomial with Newton polygon $\Delta_{5}$ defines a plane cubic which admits two tangent lines intersecting at some point on a curve, but the Plücker formulas show that this is impossible for a cuspidal cubic.

4.3. Amoebas and tropicalizations of 1-cuspidal curves. The dimension of the stratum of curves with a cusp in $\Lambda(\Delta)$ is $r=\left|\mathbb{Z}^{2} \cap \Delta\right|-3$. We pick $r$ distinct generic points $\boldsymbol{x}_{1}, \ldots, \boldsymbol{x}_{r} \in \mathbb{Q}^{2}$ and points $\boldsymbol{p}_{1}, \ldots, \boldsymbol{p}_{r} \in\left(\mathbb{K}^{*}\right)^{2}$ so that $\operatorname{Val}\left(\boldsymbol{p}_{i}\right)=\boldsymbol{x}_{i}, i=1, \ldots, r$.

The exponents of $t$ in the coefficients of all the polynomials $f \in \mathbb{K}[x, y]$ such that the cuspidal curves in $\Lambda_{\mathbb{K}}(\Delta)$ defined by them pass through $\boldsymbol{p}_{1}, \ldots, \boldsymbol{p}_{r}$ have a common denominator. A parameter change $t \mapsto t^{M}$ with a suitable natural $M$ makes all these exponents integral and the convex piecewise linear function $\nu_{f}: \Delta \rightarrow \mathbb{R}$ integral-valued at integral points.

We claim that the images of the 1 -cuspidal curves in $\Lambda_{\mathbb{K}}(\Delta)$ that pass through $\boldsymbol{p}_{1}, \ldots$, $\boldsymbol{p}_{r}$ are 1-cuspidal amoebas passing through $\boldsymbol{x}_{1}, \ldots, \boldsymbol{x}_{r}$. Indeed, using Lemmas 3.5, 4.3(i), and 4.2 (vi), it is easy to show that the amoebas of rank exceeding $\left|\mathbb{Z}^{2} \cap \Delta\right|-3$, as well as the non-1-cuspidal amoebas of rank $\left|\mathbb{Z}^{2} \cap \Delta\right|-3$ can lift only to nodal curves.

Furthermore, the polynomials in $\mathcal{T}(f)$ corresponding to the polygons in $S_{f}$ that are Aff $\left(\mathbb{Z}^{2}\right)$-equivalent to those shown in Figure $4(\mathrm{a}),(\mathrm{b}),(\mathrm{c}),(\mathrm{d})$ must be as described in Lemma $4.2(\mathrm{i})-(\mathrm{v})$, and the polynomials in $\mathcal{T}(f)$ corresponding to the triangles without interior integral points must be as described in Lemma 3.5. If $S_{f}$ contains a polygon Aff $\left(\mathbb{Z}^{2}\right)$-equivalent to one of those shown in Figure 4(c), (d), then there is an edge $\sigma=$ $\Delta_{k} \cap \Delta$ and a point $z \in \operatorname{Tor}(\sigma) \cap C_{k} \cap C_{l}$ such that $\left(C_{k} \cdot \operatorname{Tor}(\sigma)\right)_{z}=\left(C_{l} \cdot \operatorname{Tor}(\sigma)\right)_{z} \geq 2$, and thus, we can construct a $z$-refinement of the tropicalization of $f$ as was explained in Subsection 3.5. Possible refinements of the tropicalization of $f$ are described in the following statements.

Lemma 4.3. Under the above notation and definitions, let $z \in \operatorname{Tor}(\sigma) \cap C_{k} \cap C_{l}$.

(i) If $C_{k}$ and $C_{l}$ are nonsingular at $z,\left(C_{k} \cdot \operatorname{Tor}(\sigma)\right)_{z}=3$, and $z$ bears a cusp $A_{2}$, then $\mathcal{T}_{z}(f)$ consists of one polynomial having Newton polygon $\Delta_{z}$ as shown in Figure 5(a) and defining an elliptic curve with one cusp in $\operatorname{Tor}\left(\Delta_{z}\right)$.

(ii) If $C_{k}$ has a node at $z, C_{l}$ is nonsingular at $z,\left(C_{k} \cdot \operatorname{Tor}(\sigma)\right)_{z}=2$, and $z$ bears a cusp $A_{2}$, then $\mathcal{T}_{z}(f)$ consists of one polynomial having Newton polygon $\Delta_{z}$ as shown in Figure 5 (b) and defining a rational curve with one cusp in $\operatorname{Tor}\left(\Delta_{z}\right)$. 


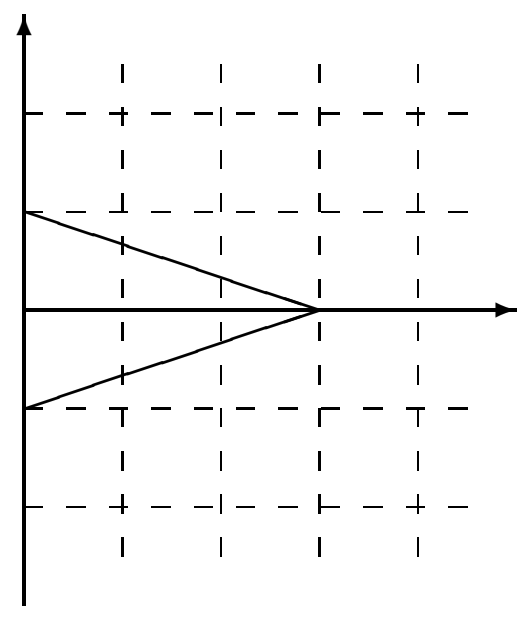

(a)

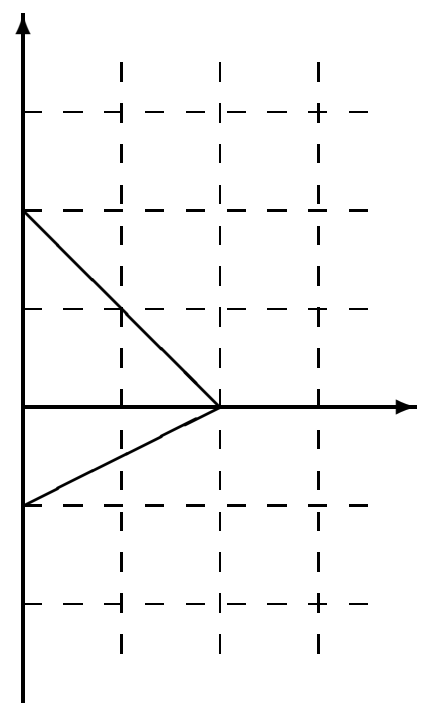

(b)

FiguRE 5. Newton polygons for deformation patterns

Proof. We show that in each case $\mathcal{T}_{z}(f)$ consists of one polynomial and describe these polynomials in Lemma 4.4 below.

The proof proceeds in the same way as the proof of Lemma 3.10. As an example, we treat situation (ii).

Besides the vertices of $\Delta_{z}$, only the points $(0,0)$ and $(0,1)$ may serve as vertices of the subdivision $S_{f(z)}$. Observe that the possible intersections of the curves defined by $\mathcal{T}_{z}(f)$ with $\operatorname{Tor}([(0,0),(2,0)])$ are transversal, because the coefficient of $x$ is zero by construction. Consequently, $z$ may only bear singular points that come from singularities in $\left(\mathbb{C}^{*}\right)^{2}$ of curves defined by polynomials with Newton polygon $\operatorname{conv}\{(0,0),(2,0),(0,2)\}$ or $\operatorname{conv}\{(0,-1),(0,1),(2,0)\}$, which are at most nodes.

Lemma 4.4. (i) There exist exactly two polynomials with Newton polygon $\Delta^{\prime}$ as shown in Figure [5) and such that they have prescribed coefficients at the vertices of $\Delta^{\prime}$ and the zero coefficient of $x^{2}$ and define curves in $\operatorname{Tor}\left(\Delta^{\prime}\right)$ with a cusp as their only singularity.

(ii) There exist exactly three polynomials with Newton polygon $\Delta^{\prime \prime}$ as shown in Figure 5 (b) and such that they have prescribed coefficients at the vertices of $\Delta^{\prime \prime}$ and the zero coefficient of $x^{2}$ and define curves in $\operatorname{Tor}\left(\Delta^{\prime \prime}\right)$ with a cusp as their only singularity.

Proof. This is checked by direct computation. As an example, we consider the second case.

After a suitable coordinate change, we reduce the question to the study of polynomials

$$
F(x, y)=y^{3}+y x^{2}+1+a y+b y^{2}, \quad a, b \in \mathbb{C} .
$$

The system $F(x, y)=F_{x}(x, y)=F_{y}(x, y)=0$ reduces in $\mathbb{C}^{2}$ to the system

$$
x=0, \quad 3 y^{2}+2 b y+a=0, \quad y^{3}+b y^{2}+a y+1=0,
$$

which must have a solution of multiplicity 2 , so that $y^{3}+b y^{2}+a y+1=(y+\alpha)^{3}, \alpha^{3}=1$, and the claim follows. 
4.4. Restoring a cuspidal curve out of the tropical data. We follow the argument of Subsection 3.7 .

Let $\mathcal{Q}_{\Delta}\left(A_{2}\right)$ denote the set of quadruples $(A, S, F, R)$, where

- $A \in \mathcal{A}(\Delta)$ is a 1-cuspidal amoeba, $S: \Delta=\Delta_{1}, \ldots, \Delta_{N}$ is a subdivision of $\Delta$ dual to $A$, and $F, R$ are collections of polynomials in $\mathbb{C}[x, y]$, which together are defined up to multiplication by the same nonzero (complex) constant;

- $F=\left(f_{1}, \ldots, f_{N}\right)$, where $f_{i}$ is a polynomial with Newton polygon $\Delta_{i}, i=$ $1, \ldots, N$, and such that if $\Delta_{i}$ is a triangle without interior integral points, then $f_{i}$ defines a rational curve in $\operatorname{Tor}\left(\Delta_{i}\right)$ as described in Lemma 3.5. if $\Delta_{i}$ is a triangle $\operatorname{Aff}\left(\mathbb{Z}^{2}\right)$-equivalent to that shown in Figure $4(\mathrm{~b}),(\mathrm{c})$, then $f_{i}$ defines a curve in $\operatorname{Tor}\left(\Delta_{i}\right)$ as described in Lemma 4.2 (ii), (iii); if $\Delta_{i}$ is a quadrangle $\operatorname{Aff}\left(\mathbb{Z}^{2}\right)$ equivalent to that shown in Figure 4 (a), (d), then $f_{i}$ defines a curve in $\operatorname{Tor}\left(\Delta_{i}\right)$ as described in Lemma 4.2(iv), (v), and finally, for any common edge $\sigma=\Delta_{i} \cap \Delta_{j}$ the truncations $f_{i}^{\sigma}$ and $f_{j}^{\sigma}$ coincide;

- $R$ is a collection of deformation patterns compatible with $F$ as described in Remarks 3.8 and 3.11 .

Let $(A, S, F, R) \in \mathcal{Q}_{\Delta}\left(A_{2}\right)$, and let $\boldsymbol{x}_{1}, \ldots, \boldsymbol{x}_{r} \in A$. These points lie on $r$ distinct edges of $A$ corresponding to some $r$ edges of $S$, and they impose conditions (3.7.13) on the values of the function $\nu: \Delta \rightarrow \mathbb{R}$. Since $\boldsymbol{x}_{1}, \ldots, \boldsymbol{x}_{r}$ are generic, system (3.7.13) is independent. A quadrangle that may appear in $S$ imposes one linear condition on the values of $\nu$ at the vertices; in the case of the shape shown in Figure 4 (a), this condition reads

$$
3 \nu(1,0)+\nu(1,2)=2 \nu(0,1)+2 \nu(2,0),
$$

and in the case of Figure 4 (d) we have

$$
\nu(0,0)+2 \nu(1,1)=2 \nu(0,1)+\nu(2,0) .
$$

Adding the latter condition to 3.7.13), we obtain a system of $|V(S)|-1$ independent equations, which determines the values of $\nu$ at $V(S)$ uniquely up to a shift.

We seek a polynomial $f \in \mathbb{K}[x, y]$ that defines a curve in $\Sigma_{\Delta}\left(A_{2}\right)$ in the form (3.7.15). As at Step 2 in Subsection 3.7, conditions (3.7.16) take the form (3.7.17). In the case of a triangular subdivision $S$, the latter system determines the coefficients of $f_{1}, \ldots, f_{N}$ at $V(S)$, as well as the truncations of $f_{1}, \ldots, f_{N}$ on the edges $\sigma_{1}, \ldots, \sigma_{r}$, uniquely up to proportionality. If $S$ contains a quadrangle, then we supply system (3.7.17) with equation (4.2.18) or (4.2.19), which together produce $w\left(A, \boldsymbol{x}_{1}, \ldots, \boldsymbol{x}_{r}\right)$ collections of the coefficients of $f_{1}, \ldots, f_{N}$ at $V(S)$ and truncations to $\sigma_{1}, \ldots \sigma_{r}$ (up to proportionality). Finally, using Lemmas 4.2 and 4.4, we see that for a given 1-cuspidal amoeba $A \in \mathcal{A}(\Delta), A \supset$ $\left\{\boldsymbol{x}_{1}, \ldots, \boldsymbol{x}_{r}\right\}$, there are $W\left(A, \boldsymbol{x}_{1}, \ldots, \boldsymbol{x}_{r}\right) \prod_{i=1}^{r}\left|\sigma_{i}\right|^{-1}$ quadruples $(A, S, F, R) \in \mathcal{Q}_{\Delta}\left(A_{2}\right)$ that may serve as tropicalizations of polynomials $f \in \mathbb{K}[x, y]$ having Newton polygon $\Delta$ and defining curves $C \in \Sigma_{\Delta}\left(A_{2}\right)$ that pass through $\boldsymbol{p}_{1}, \ldots, \boldsymbol{p}_{r}$.

Lemma 4.5. In the above notation, for given $\boldsymbol{x}_{1}, \ldots, \boldsymbol{x}_{r} \in \mathbb{Q}^{2}, \boldsymbol{p}_{1}, \ldots, \boldsymbol{p}_{r} \in\left(\mathbb{K}^{*}\right)^{2}$ and a compatible $(A, S, F, R) \in \mathcal{Q}_{\Delta}\left(A_{2}\right)$, the polynomials $f \in \mathbb{K}[x, y]$ with Newton polygon $\Delta$ that tropicalize into $(A, S, F, R)$ define exactly $\prod_{i=1}^{r}\left|\sigma_{i}\right|$ curves in $\Lambda_{\mathbb{K}}(\Delta)$ that have one cusp and pass through $\boldsymbol{p}_{1}, \ldots, \boldsymbol{p}_{r}$.

The proof of Lemma 4.5) is completely similar to that of Lemma 3.12 (see Subsection [5.4), and we omit it. 


\section{§5. PatchWorking Singular algebraic CURVes}

5.1. Initial data for patchworking. Let $\Delta \subset \mathbb{R}^{2}$ be a nondegenerate convex lattice polygon, and let $S: \Delta=\Delta_{1} \cup \cdots \cup \Delta_{N}$ be its subdivision into convex lattice polygons determined by a convex piecewise linear function $\nu: \Delta \rightarrow \mathbb{R}$ such that $\nu\left(\mathbb{Z}^{2}\right) \subset \mathbb{Z}$.

Suppose $a_{i j} \in \mathbb{C},(i, j) \in \Delta \cap \mathbb{Z}^{2}$, are such that $a_{i j} \neq 0$ for each vertex $(i, j)$ of all the polygons $\Delta_{1}, \ldots, \Delta_{N}$. Then we introduce the polynomials

$$
f_{k}(x, y)=\sum_{(i, j) \in \Delta_{k} \cap \mathbb{Z}^{2}} a_{i j} x^{i} y^{j}, \quad k=1, \ldots, N,
$$

and the curves $C_{k}=\left\{f_{k}=0\right\} \subset \operatorname{Tor}\left(\Delta_{k}\right), k=1, \ldots, N$, on which we impose the following conditions.

(A) For any $k=1, \ldots, N$, each multiple component of $C_{k}$ (if it exists) is defined by a binomial; it crosses any other component of $C_{k}$ transversally, only at nonsingular points, and not on $\operatorname{Tor}\left(\partial \Delta_{k}\right)$.

(B) For any edge $\sigma \subset \partial \Delta, \sigma \subset \Delta_{k}, 1 \leq k \leq N$, the curve $C_{k}$ is nonsingular along $\operatorname{Tor}(\sigma)$ and crosses Tor $(\sigma)$ transversally.

(C) If $\sigma$ is an edge of $\Delta_{k}, 1 \leq k \leq N$, and $z \in \operatorname{Tor}(\sigma) \cap C_{k}$ is an isolated singular point of $C_{k}$, then the germ $\left(C_{k}, z\right)$ is topologically equivalent to $y^{m(k, z)}+x^{m}=0$, where the $y$-axis stands for $\operatorname{Tor}(\sigma)$.

Now we introduce additional polynomials that will play the role of deformation patterns, as defined in Remarks 3.8 and 3.11 .

Consider all the triples $(k, \sigma, z)$ in which $1 \leq k \leq N, \sigma \not \subset \partial \Delta$ is an edge of $\Delta_{k}$, $z \in \operatorname{Tor}(\sigma) \cap C_{k}$, and $\left(C_{k} \cdot \operatorname{Tor}(\sigma)\right)_{z}=m \geq 2$. We introduce the following equivalence of triples: (i) $(k, \sigma, z) \sim(l, \sigma, z)$ if $\sigma=\Delta_{k} \cap \Delta_{l}$, and (ii) $(k, \sigma, z) \sim\left(k, \sigma^{\prime}, z^{\prime}\right)$ if $\sigma, \sigma^{\prime}$ are parallel sides of $\Delta_{k}$ and $z, z^{\prime}$ belong to one and the same multiple component of $C_{k}$. The transitive extension of this equivalence distributes the triples into disjoint classes. We denote the set of equivalence classes by $\Pi$. In fact, a pair of points $z, z^{\prime}$ in equivalent triples $(k, \sigma, z),\left(l, \sigma^{\prime}, z^{\prime}\right)$ determines an element of $\Pi$ uniquely, and we write simply $\left(z, z^{\prime}\right) \in \Pi$.

With any element of $\Pi$ we associate a deformation pattern. Namely, in any class there are exactly two triples $(k, \sigma, z),\left(l, \sigma^{\prime}, z^{\prime}\right)$ with coinciding or parallel edges $\sigma, \sigma^{\prime}$ and with isolated singular (or nonsingular) points $z, z^{\prime}$ of the curves $C_{k}, C_{l}$, respectively. As required in the above property $(\mathrm{C})$, in some local coordinates in neighborhoods of $z$ and $z^{\prime}$ the curves $C_{k}$ and $C_{l}$ are defined by

$$
\sum_{i m(k, z)+j m \geq m m(k, z)} \alpha_{i j} x^{i} y^{j}=0, \quad \sum_{i m\left(l, z^{\prime}\right)+j m \geq m m\left(l, z^{\prime}\right)} \beta_{i j} x^{i} y^{j}=0,
$$

respectively, with $\alpha_{m 0}=\beta_{m 0}$ and with nondegenerate homogeneous polynomials

$$
\begin{aligned}
\varphi_{z}^{(k)}(x, y) & =\sum_{i m(k, z)+j m=m m(k, z)} \alpha_{i j} x^{i} y^{j}, \\
\varphi_{z^{\prime}}^{(l)}(x, y) & =\sum_{i m\left(l, z^{\prime}\right)+j m=m m\left(l, z^{\prime}\right)} \beta_{i j} x^{i} y^{j} .
\end{aligned}
$$

A deformation pattern associated with a chosen class of triples is a curve $C_{z, z^{\prime}} \subset$ $\operatorname{Tor}\left(\Delta_{z, z^{\prime}}\right), \Delta_{z, z^{\prime}}=\operatorname{conv}\left\{(m, 0),(0, m(k, z)),\left(0,-m\left(l, z^{\prime}\right)\right)\right\}$, defined by a polynomial $F_{z, z^{\prime}}(x, y)$ with Newton triangle $\Delta_{z, z^{\prime}}$ and truncations $\varphi_{z}^{(k)}(x, y), \varphi_{z^{\prime}}^{(l)}\left(x, y^{-1}\right)$ on the edges $\left[(m, 0),(0, m(k, z)],\left[(m, 0),\left(0,-m\left(l, z^{\prime}\right)\right)\right]\right.$, respectively. 
5.2. Transversality. Transversality of equisingular strata provides sufficient conditions for the patchworking (cf. [25, 26]).

Let $\mathcal{S}$ be a topological or (contact) analytic equivalence of isolated planar curve singular points. We intend to define $\mathcal{S}$-transversality for triples $\left(\Delta_{k}, \Delta_{k}^{-}, C_{k}\right), 1 \leq k \leq N$, where $\Delta_{k}^{-}$is a connected (or empty) union of some edges of $\Delta_{k}$, and for deformation patterns.

Pick a triple $\left(\Delta_{k}, \Delta_{k}^{-}, C_{k}\right), 1 \leq k \leq N$.

If $z \in C_{k} \cap\left(\mathbb{C}^{*}\right)^{2}$ is an isolated singular point, we denote by $M^{\mathcal{S}}\left(C_{k}, z\right)$ the germ at $C_{k}$ of the $\mathcal{S}$-equisingular stratum of $\left(C_{k}, z\right)$ in $\Lambda\left(\Delta_{k}\right)$. The (projective) Zariski tangent space to $M^{\mathcal{S}}\left(C_{k}, z\right)$ at $C_{k}$ is formed by the curves $\{g=0\}, g \in \Lambda\left(\Delta_{k}\right)$, with $g \in I^{\mathcal{S}}\left(C_{k}, z\right) \subset$ $\mathcal{O}_{\operatorname{Tor}\left(\Delta_{k}\right), z}$, where $I^{\mathcal{S}}\left(C_{k}, z\right)$ is the equisingular ideal (see [5, 34]) or the Tyurina ideal $\left\langle g, g_{x}, g_{y}\right\rangle$, according to whether $\mathcal{S}$ is the topological or analytic equivalence.

Let $z \in C_{k} \cap \operatorname{Tor}(\sigma)$ be a nonsingular or singular isolated point of $C_{k}$, where $\sigma$ is an edge of $\Delta_{k}$, and let $x^{\prime \prime}, y^{\prime \prime}$ be local coordinates in a neighborhood of $z$ in $\operatorname{Tor}\left(\Delta_{k}\right)$, as was introduced in Subsection 5.1. The ideals

$$
\begin{aligned}
& I_{0}^{\mathrm{sqh}}\left(C_{k}, z\right)=\left\{g \in \mathcal{O}_{\operatorname{Tor}\left(\Delta_{k}\right), z}: g=\sum_{i m(k, z)+j m \geq m m(k, z)} b_{i j}\left(x^{\prime \prime}\right)^{i}\left(y^{\prime \prime}\right)^{j}\right\}, \\
& I^{\mathrm{sqh}}\left(C_{k}, z\right)=I_{0}^{\mathrm{sqh}}\left(C_{k}, z\right)+\left\langle\frac{\partial f^{\prime \prime}}{\partial x^{\prime \prime}}\right\rangle
\end{aligned}
$$

naturally determine the linear subsystems $M_{0}^{\mathrm{sqh}}\left(C_{k}, z\right)$ and $M^{\mathrm{sqh}}\left(C_{k}, z\right)$ in $\Lambda\left(\Delta_{k}\right)$, respectively.

Let $z \in C_{k} \cap\left(\mathbb{C}^{*}\right)^{2}$ be an intersection point of two distinct components $\left\{g^{\prime}=0\right\}$ and $\left\{g^{\prime \prime}=0\right\}$ of $C_{k}$ having multiplicities $m^{\prime}$ and $m^{\prime \prime}$, respectively, with $m^{\prime}+m^{\prime \prime}>2$. We denote by $M^{e g}\left(C_{k}, z\right)$ the closure of the germ at $C_{k}$ of the family of curves $C \in \Lambda\left(\Delta_{k}\right)$ having $m^{\prime} m^{\prime \prime}$ nodes in a neighborhood of $z$. The (projective) Zariski tangent space to $M^{e g}\left(C_{k}, z\right)$ at $C_{k}$ is formed by the curves $\{g=0\}, g \in \Lambda\left(\Delta_{k}\right)$, with $g \in I^{e g}\left(C_{k}, z\right):=$ $\left\langle\left(g^{\prime}\right)^{m^{\prime}},\left(g^{\prime \prime}\right)^{m^{\prime \prime}}\right\rangle \subset \mathcal{O}_{\operatorname{Tor}\left(\Delta_{k}\right), z}$. Indeed, in a neighborhood of $z$, the curves $C \in M^{e g}\left(C_{k}, z\right)$ are unions of $m^{\prime}+m^{\prime \prime}$ disks (counting multiplicities) and are represented by equations $\left(\left(g^{\prime}\right)^{m^{\prime}}+g_{1}^{\prime}\right)\left(\left(g^{\prime \prime}\right)^{m^{\prime \prime}}+g_{1}^{\prime \prime}\right)=0$ with $\left\|g_{1}^{\prime}\right\|$ and $\left\|g_{1}^{\prime \prime}\right\|$ sufficiently small; thus, the claim follows.

Definition 5.1. In the above notation, let $\Delta_{k}^{+}$be the union of the edges $\sigma$ of $\Delta_{k}$ such that $\sigma \not \subset \Delta_{k}^{-}$. The triple $\left(\Delta_{k}, \Delta_{k}^{-}, C_{k}\right)$ is said to be $\mathcal{S}$-transversal if all the germs

$$
\begin{cases}M^{\mathcal{S}}\left(C_{k}, z\right), & z \in\left(\mathbb{C}^{*}\right)^{2} \text { is an isolated singular point of } C_{k} ; \\ M^{e g}\left(C_{k}, z\right), & z \in\left(\mathbb{C}^{*}\right)^{2} \text { is an intersection point of a multiple component } \\ & \text { of } C_{k}, \text { with any other component of } C_{k} ; \\ M_{0}^{\text {sqh }}\left(C_{k}, z\right), & z \in C_{k} \cap \operatorname{Tor}\left(\Delta_{k}^{-}\right) \text {is not a nonisolated singular point; } \\ M^{\text {sqh }}\left(C_{k}, z\right), & z \in C_{k} \cap \operatorname{Tor}\left(\Delta_{k}^{+}\right) \text {is not a nonisolated singular point, }\end{cases}
$$

are smooth germs of expected dimension and intersect transversally in $\Lambda\left(\Delta_{k}\right)$.

Definition 5.2. A deformation pattern $C_{z, z^{\prime}} \subset \Delta_{z, z^{\prime}}$ is said to be $\mathcal{S}$-transversal if the triple $\left(\Delta_{z, z^{\prime}}, \Delta_{z, z^{\prime}}^{-}, C_{z, z^{\prime}}\right)$ is $\mathcal{S}$-transversal, where $\Delta_{z, z^{\prime}}^{-}$is the union of the nonvertical edges of $\Delta_{z, z^{\prime}}$.

Lemma 5.3. In the above notation, the triple $\left(\Delta_{k}, \Delta_{k}^{-}, C_{k}\right)$ is $\mathcal{S}$-transversal if

$$
H^{1}\left(\operatorname{Tor}\left(\Delta_{k}\right), \mathcal{O}_{\operatorname{Tor}\left(\Delta_{k}\right)}\left(C_{k}\right) \otimes \mathcal{J}_{Z_{k}}\right)=0
$$


where $\mathcal{J}_{Z_{k}} \subset \mathcal{O}_{\operatorname{Tor}\left(\Delta_{k}\right)}$ is the ideal sheaf of the zero-dimensional scheme $Z_{k} \subset \operatorname{Tor}\left(\Delta_{k}\right)$ defined at the points $z \in C_{k}$ mentioned in Definition 5.1 by the ideals $I^{\mathcal{S}}\left(C_{k}, z\right), I^{e g}\left(C_{k}, z\right)$, $I_{0}^{s q h}\left(C_{k}, z\right)$, and $I^{s q h}\left(C_{k}, z\right)$, respectively.

This statement immediately follows from the cohomology interpretation of transversality.

Following [25, 26, we provide an explicit numerical criterion for the $h^{1}$-vanishing (5.2.20). To formulate it, we use the topological invariants $b(C, \xi), \widetilde{b}(C, \xi)$ defined for a curve $C$ and its local branch $\xi$, and the Tyurina number $\tau(C, z)$ equal to the codimension of the Tyurina ideal in the local ring of an ambient surface. The complete definition of the invariants $b$ and $\widetilde{b}$ can be found in [25, Subsection 4.1], or in [26, $\S 4$, Definition 1]. We only recall it for some cases. If $C$ has a node, then $b(C, \xi)=0$ for both branches; if $C$ has a cusp, then $b(C, \xi)=1$; if $C$ is given locally by $\left\{x^{p r}+y^{q r}=0\right\}$, with $(p, q)=1$, then $\widetilde{b}(C, \xi)=p+q-1$.

\section{Lemma 5.4.}

(i) The $\mathcal{S}$-transversality of a triple $\left(\Delta_{k}, \Delta_{k}^{-}, C_{k}\right)$ survives if $\Delta_{k}^{-}$contains at most two edges and from $\Delta_{k}^{-}$we remove edges of length 1 .

(ii) If $C_{k}$ is irreducible, then the triple $\left(\Delta_{k}, \Delta_{k}^{-}, C_{k}\right)$ is transversal with respect to the topological equivalence of singular points provided that

$$
\sum^{\prime} b\left(C_{k}, \xi\right)+\sum^{\prime \prime} \widetilde{b}\left(C_{k}, \mathcal{Q}\right)+\sum^{\prime \prime \prime}\left(\left(C_{k} \cdot \operatorname{Tor}(\sigma)\right)_{z}-\varepsilon\right)<\sum_{\sigma \subset \partial \Delta_{k}}\left(C_{k} \cdot \operatorname{Tor}(\sigma)\right),
$$

where $\sum^{\prime}$ ranges over all local branches $\xi$ of $C_{k}$ centered at the points $z \in$ $\operatorname{Sing}\left(C_{k}\right) \cap\left(\mathbb{C}^{*}\right)^{2}, \sum^{\prime \prime}$ ranges over all local branches $\mathcal{Q}$ of $C_{k}$ centered at the points $z \in \operatorname{Sing}\left(C_{k}\right) \cap \operatorname{Tor}\left(\partial \Delta_{k}\right)$, and $\sum^{\prime \prime \prime}$ ranges over all nonsingular points $z$ of $C_{k}$ on $\operatorname{Tor}\left(\partial \Delta_{k}\right)$, with $\varepsilon=0$ if $\sigma \subset \Delta_{k}^{-}$and $\varepsilon=1$ otherwise.

(iii) If $C_{k}$ is irreducible, then the triple $\left(\Delta_{k}, \Delta_{k}^{-}, C_{k}\right)$ is transversal with respect to the analytic equivalence of singular points provided that

$$
\sum^{\prime}\left(\tau\left(C_{k}, z\right)-1\right)+\sum^{\prime \prime} \widetilde{b}\left(C_{k}, \mathcal{Q}\right)+\sum^{\prime \prime \prime}\left(\left(C_{k} \cdot \operatorname{Tor}(\sigma)\right)_{z}-\varepsilon\right)<\sum_{\sigma \subset \partial \Delta_{k}}\left(C_{k} \cdot \operatorname{Tor}(\sigma)\right),
$$

where $\sum^{\prime}$ ranges over all $z \in \operatorname{Sing}\left(C_{k}\right) \cap\left(\mathbb{C}^{*}\right)^{2}$, and $\sum^{\prime \prime}$ and $\sum^{\prime \prime \prime}$ are as above.

(iv) If $C_{k}$ is reduced and reducible, then the triple $\left(\Delta_{k}, \Delta_{k}^{-}, C_{k}\right)$ is transversal with respect to the topological equivalence of singular points provided that, for any irreducible component $C$ of $C_{k}$,

$$
\sum^{\prime} b\left(C_{k}, \xi\right)+\sum^{\prime \prime} \widetilde{b}\left(C_{k}, \mathcal{Q}\right)+\sum^{\prime \prime \prime}\left((C \cdot \operatorname{Tor}(\sigma))_{z}-\varepsilon\right)<\sum_{\sigma \subset \partial \Delta_{k}}(C \cdot \operatorname{Tor}(\sigma)),
$$

where $\sum^{\prime}$ ranges over all local branches $\xi$ of $C$ centered at the points $z \in \operatorname{Sing}\left(C_{k}\right)$ $\cap\left(\mathbb{C}^{*}\right)^{2}, \sum^{\prime \prime}$ ranges over all local branches $\mathcal{Q}$ of $C$ centered at the points $z \in$ $\operatorname{Sing}\left(C_{k}\right) \cap \operatorname{Tor}\left(\partial \Delta_{k}\right)$, and $\sum^{\prime \prime \prime}$ ranges over all nonsingular points $z$ of $C_{k}$ on $C \cap \operatorname{Tor}(\sigma), \sigma \subset \partial \Delta_{k}$, with $\varepsilon=0$ if $\sigma \subset \Delta_{k}^{-}$and $\varepsilon=1$ otherwise.

(v) If $C_{k}$ is nonreduced, then the triple $\left(\Delta_{k}, \Delta_{k}^{-}, C_{k}\right)$ is transversal with respect to the topological equivalence of singular points provided that any component of $C_{k}$ that is not defined by a binomial satisfies (5.2.21), and any component of $C_{k}$ defined by a binomial crosses $\operatorname{Tor}\left(\Delta_{k}^{-}\right)$at one point at most and crosses the reduced union of all the other components of $C_{k}$ transversally at nonsingular points only. 
Lemma 5.5. In the notation of Subsection 5.1 and under Definition 5.2

(i) an irreducible deformation pattern $C_{z, z^{\prime}}$ is transversal with respect to the topological equivalence of singular points if

$$
\sum_{w \in \operatorname{Sing}\left(C_{z, z^{\prime}}\right) \cap \mathbb{C}^{2}} b\left(C_{z, z^{\prime}}, w\right)<\#\left(\mathbb{Z} \cap\left(-m\left(l, z^{\prime}\right), m(k, z)\right)\right)+\varepsilon_{0}
$$

and is transversal with respect to the analytic equivalence of singular points if

$$
\sum_{w \in \operatorname{Sing}\left(C_{z, z^{\prime}}\right) \cap \mathbb{C}^{2}}\left(\tau\left(C_{z, z^{\prime}}, w\right)-1\right)<\#\left(\mathbb{Z} \cap\left(-m\left(l, z^{\prime}\right), m(k, z)\right)\right)+\varepsilon_{0},
$$

where $\varepsilon_{0}$ is the number of edges of length 1 in $\Delta_{z, z^{\prime}}^{-}$;

(ii) a reducible deformation pattern $C_{z, z^{\prime}}$ is transversal with respect to the topological equivalence of singular points if for any irreducible component $C$ of $C_{z, z^{\prime}}$ we have

$$
\sum_{\xi} b\left(C_{z, z^{\prime}}, \xi\right)<\left(C \cdot \operatorname{Tor}\left(\partial \Delta_{z, z^{\prime}}\right)\right)-\left(C \cdot \operatorname{Tor}\left(\Delta_{z, z^{\prime}}^{-}\right)\right)+\varepsilon_{0}(C),
$$

where $\xi$ ranges over all local branches of $C$ centered in $\operatorname{Sing}\left(C_{z, z^{\prime}}\right) \cap \mathbb{C}^{2}$, and $\varepsilon_{0}(C)$ is the number of edges of length 1 in $\Delta_{z, z^{\prime}}^{-}$.

Proof. Both Lemmas 5.4 and 5.5 are slightly modified particular cases of Theorem 4.1 in [25, and we shall only explain the modifications.

To obtain $\varepsilon_{0}$, or, more generally, to remove edges of length 1 from $\Delta_{k}^{-}$, we notice that the $\mathcal{S}$-transversality of the triad $\left(\Delta_{k}, \widetilde{\Delta}_{k}^{-}, C_{k}\right)$ means that in the space $\mathcal{P}\left(\Delta_{k}\right)$ of polynomials with Newton polygon $\Delta_{k}$, the corresponding $\mathcal{S}$-equisingular stratum is smooth and transversally intersects the (affine) subspace of polynomials with fixed coefficients at the integral points in $\widetilde{\Delta}_{k}^{-}$. The action of $\left(\mathbb{C}^{*}\right)^{3}$ on $\mathcal{P}\left(\Delta_{k}\right)$, defined as $\left(\lambda_{0}, \lambda_{1}, \lambda_{2}\right) \cdot F(x, y)=\lambda_{0} F\left(\lambda_{1} x, \lambda_{2} y\right)$, arbitrarily varies the coefficients, corresponding to integral points in the edges of length 1 in $\Delta_{k}^{-} \backslash \widetilde{\Delta}_{k}^{-}$, whereas the coefficients at the integral points in $\widetilde{\Delta}_{k}^{-}$stay fixed. Since the $\mathcal{S}$-equisingular stratum in question is invariant with respect to this action, we conclude that it transversally intersects the subspace of polynomials with fixed coefficients at the integral points in $\Delta_{k}^{-}$.

In case (v) in Lemma 5.4 relation (5.2.20) is deduced by successively eliminating the components of $C_{k}$ defined by binomials with the help of the Horace method [9], and then applying statement (iii) to the remaining part of the curve.

5.3. Patchworking theorem. Suppose we are given the data introduced in Subsection 5.1 namely, a subdivision $S: \Delta=\Delta_{1} \cup \cdots \cup \Delta_{N}$ induced by a function $\nu: \Delta \rightarrow \mathbb{R}$, an amoeba $A$, polynomials $f_{1}, \ldots, f_{N}$, and deformation patterns defined by polynomials $f_{z, \widetilde{z}}$. Let $\mathcal{G}$ be the set of orientations of the amoeba $A$ (viewed as a graph) that have no oriented cycles and obey the following requirements. For $\Gamma \in \mathcal{G}$, let $\Delta_{k}^{-}(\Gamma)$ denote the union of the edges of $\Delta_{k}$ that correspond to the arcs of $A \Gamma$-oriented inside $\Delta_{k}$. We assume that $\Delta_{k}^{-}(\Gamma)$ is connected for any $k=1, \ldots, N$ and that any two arcs of $A$ having a common vertex and lying on a straight line are cooriented.

Theorem 5. Under the assumptions of Subsection 5.1, suppose that all the given deformation patterns are $\mathcal{S}$-transversal, and there is $\Gamma \in \mathcal{G}$ such that every triple $\left(\Delta_{k}, \Delta_{k}^{-}, C_{k}\right)$ is $\mathcal{S}$-transversal, $k=1, \ldots, N$. Then there exists a polynomial $f \in \mathbb{K}[x, y]$ with the following properties: its Newton polygon is $\Delta$; its refined tropicalization consists of the given data $\nu, S, f_{1}, \ldots, f_{N}$ and the given deformation patterns; it defines a family of reduced curves $C^{(t)} \subset \operatorname{Tor}(\Delta), t \neq 0$, such that there is an $\mathcal{S}$-equivalent one-to-one correspon- 
dence between $\operatorname{Sing}\left(C^{(t)}\right)$ and the disjoint union of

- the sets of isolated singular points of all the curves $C_{k}$ in $\left(\mathbb{C}^{*}\right)^{2}, k=1, \ldots, N$,

- the sets $\operatorname{Sing}\left(C_{z, \tilde{z})} \cap \mathbb{C}^{2},\{z, \widetilde{z}\} \in \Pi\right.$,

- the set of $\sum_{k=1}^{N} \sum_{z} \operatorname{dim} \mathcal{O}_{\mathbb{C}^{2}, z} / I^{e g}\left(C_{k}, z\right)$ nodes, where $z$ runs over all points in $C_{k}$, belonging to the intersections of a multiple component of $C_{k}$ with any other component of $C_{k}$.

Furthermore, take any set $B \subset V(S)$ such that, for every $k=1, \ldots, N$, either $\left|B \cap \Delta_{k}\right| \leq 3$, or $B \cap \Delta_{k} \subset \Delta_{k}^{-}$. Then a family of polynomials as above can be described by the relations

$$
\begin{gathered}
f(x, y)=\sum_{(i, j) \in \Delta}\left(a_{i j}+c_{i j}\right) x^{i} y^{j} t^{\nu(i, j)}, \\
\left\{\begin{array}{l}
c_{i j}=c_{i j}(t) \in \mathbb{K}, \quad c_{i j}(0)=0, \quad(i, j) \in \Delta, \\
c_{i j}(t)=\Phi_{i j}^{B}\left(\left\{c_{k l}(t),(k, l) \in B\right\}, t\right), \quad(i, j) \in \Delta \cap \mathbb{Z}^{2} \backslash B,
\end{array}\right.
\end{gathered}
$$

with certain complex analytic functions $\Phi_{i j}^{B},(i, j) \in \Delta \cap \mathbb{Z}^{2} \backslash B$.

We omit the proof, which is a routine adaptation of the proofs of similar patchworking theorems in [25, 26] (for the details we refer the reader to [27]).

5.4. Proof of Lemma 3.12, Let $(A, S, F, R) \in \mathcal{Q}\left(n A_{1}\right)$. We take any vector $\zeta \in$ $\mathbb{R}^{2} \backslash\{0\}$ not parallel to any of the edges of $S$ and orient the arcs of $A$ so that they form acute angles with $\zeta$. This gives rise to an orientation $\Gamma$ of $A$ that meets the requirements of Theorem 5. Furthermore, Lemmas 5.4 and 5.5 imply that the deformation patterns $R$ and all the triples $\left(\Delta_{k}, \Delta_{k}^{-}, C_{k}\right)$ are transversal. For example, if $\Delta_{k}, 1 \leq k \leq N$, is a triangle, the inequality of Lemma $5.4(\mathrm{i})$, which serves as a transversality criterion, is fulfilled, because the binvariant vanishes for nodes, $C_{k}$ is nonsingular along $\operatorname{Tor}\left(\partial \Delta_{k}\right)$, and $\varepsilon=1$ for all edges in $\Delta_{k}^{+} \neq \varnothing$. Thus, Theorem 5 applies, and the set $B$ can be chosen as follows. For any parallelogram $\Delta_{k}$ the set $\Delta_{k}^{+}$is the union of two neighboring edges. Then we take $V(S)$ and remove all the interior vertices of $\Delta_{k}^{+}$for all parallelograms $\Delta_{k}$.

Notice that $|B|=r+1$; that is, formulas (5.3.22) and (5.3.23) describe all the polynomials $f \in \mathbb{K}[x, y]$ with Newton polygon $\Delta$ that define $n$-nodal curves in $\Lambda_{K}(\Delta)$ and tropicalize into $(A, S, F, R)$.

From (5.3.23) we separate the equations for $c_{i j},(i, j) \in V(S) \backslash B$. Namely, let $\omega_{1, k}$, $\omega_{2, k}, \omega_{3, k}, \omega_{4, k}$ be the vertices (listed clockwise) of a parallelogram $\Delta_{k}$, and let $\omega_{1, k} \notin \Delta_{k}^{-}$. Then an equation for $M^{e g}\left(C_{k}\right)$ (see Subsection 5.2) involving the coefficients at the vertices of $\Delta_{k}$ can be written as

$$
\left(a_{\omega_{1, k}}+c_{\omega_{1, k}}\right)\left(a_{\omega_{3, k}}+c_{\omega_{3, k}}\right)-\left(a_{\omega_{2, k}}+c_{\omega_{2, k}}\right)\left(a_{\omega_{4, k}}+c_{\omega_{4, k}}\right)=O(t),
$$

where $O(t)$ includes the terms with $t$ to a positive power. Since

$$
a_{\omega_{1, k}} a_{\omega 3, k}-a_{\omega_{2, k}} a_{\omega_{4, k}}=0,
$$

we can rewrite (5.3.23) in the form

$$
\begin{array}{r}
c_{i j}=\Phi_{i j}\left(\left\{c_{\omega}: \omega \in V(S)\right\}\right), \quad(i, j) \in \Delta \backslash V(S), \\
c_{\omega_{1, k}} a_{\omega_{3, k}}+c_{\omega_{3, k}} a_{\omega_{1, k}}-c_{\omega_{2, k}} a_{\omega_{4, k}}-c_{\omega_{4, k}} a_{\omega_{2, k}}=O(t), \\
1 \leq k \leq N,\left|V\left(\Delta_{k}\right)\right|=4 .
\end{array}
$$

Consider the equations $f\left(\boldsymbol{p}_{1}\right)=\cdots=f\left(\boldsymbol{p}_{r}\right)=0$. Suppose $\boldsymbol{x}_{s}=\operatorname{Val}\left(\boldsymbol{p}_{s}\right)$ corresponds to an edge $\sigma_{s}$ of $S$. Without loss of generality, we assume that $\sigma_{s}$ lies on the horizontal coordinate axis, $\left.\nu\right|_{\sigma_{s}}=0$, and $\nu(i, j)>0$ for $(i, j) \notin \sigma_{s}$. Then $\boldsymbol{p}_{s}=\left(\xi_{s}^{0}+\xi_{s}^{1} t, \eta_{s}^{0}+\eta_{s}^{1} t\right)$, where $\xi_{s}^{0}, \eta_{s}^{0} \in \mathbb{C}^{*}, \xi_{s}^{1}, \eta_{s}^{1} \in \mathbb{K}, \operatorname{Val}\left(\xi_{s}^{1}\right), \operatorname{Val}\left(\eta_{s}^{1}\right) \leq 0$. 
Assume that $\left|\sigma_{s}\right|=1$, i.e., $\sigma_{s}=\left[\omega_{s}^{\prime}, \omega_{s}^{\prime \prime}\right], \omega_{s}^{\prime}=(i, 0), \omega_{s}^{\prime \prime}=(i+1,0)$. Then the equation $f\left(\boldsymbol{p}_{s}\right)=0$ in the form (3.7.17) reads

$$
\left(a_{\omega_{s}^{\prime}}+c_{\omega_{s}^{\prime}}\right)+\left(a_{\omega_{s}^{\prime \prime}}+c_{\omega_{s}^{\prime \prime}}\right)\left(\xi_{s}^{0}+\xi_{s}^{1} t\right)=O(t) ;
$$

since $a_{\omega_{s}^{\prime}}+a_{\omega_{s}^{\prime \prime}} \xi_{s}^{0}=0$, this reshapes to

$$
c_{\omega_{s}^{\prime}}+c_{\omega_{s}^{\prime \prime}} \xi_{s}^{0}=O(t)
$$

Suppose $\left|\sigma_{s}\right|=m \geq 2$, i.e, without loss of much generality, $\sigma_{s}=\left[\omega_{s}^{\prime}, \omega_{s}^{\prime \prime}\right], \omega_{s}^{\prime}=$ $(0,0), \omega_{s}^{\prime \prime}=(m, 0)$. We only consider the case where $\sigma_{s}$ is a common edge of two triangles $\Delta_{k}, \Delta_{l}$ (cf. Figure 2), because the situation where $\sigma_{s}$ is an edge of a parallelogram can be treated in the same way, but requires more complicated notation. Let $z=$ $\operatorname{Tor}\left(\sigma_{s}\right) \cap C_{k} \cap C_{l}$. We have

$$
f(x, y)=\sum_{i=0}^{m}\left(a_{i, 0}+c_{i, 0}\right) x^{i}+O(t)=\sum_{i=0}^{m} c_{i, 0} x^{i}+a_{m, 0}\left(x+\xi_{s}^{0}\right)^{m}+O(t) .
$$

The coordinate change $x=x^{\prime}+\xi_{s}^{0}$ takes $f(x, y)$ to the form

$$
f^{\prime}\left(x^{\prime}, y\right)=\sum_{i=0}^{m-1} c_{i, 0}^{\prime}\left(x^{\prime}\right)^{i}+\left(a_{m, 0}+c_{m, 0}\right)\left(x^{\prime}\right)^{m}+O(t),
$$

where

$$
c_{i, 0}^{\prime}=\sum_{j=i}^{m} c_{j}\left(\xi_{s}^{0}\right)^{j-i}\left(\begin{array}{l}
j \\
i
\end{array}\right), \quad i=0, \ldots, m-1 .
$$

Furthermore, we can find

$$
\tau=-\frac{c_{m-1,0}^{\prime}}{m a_{m, 0}}+O(t)+\text { h.o.t. } \in \mathbb{K},
$$

where "h.o.t." contains all monomials in $c_{\omega}, \omega \in \Delta$, of degree at least 2 and such that the coefficient of $x^{m-1}$ in the polynomial $f^{\prime \prime}\left(x^{\prime \prime}, y\right):=f^{\prime}\left(x^{\prime}+\tau, y\right)$ is zero. Then

$$
\begin{aligned}
& f^{\prime \prime}\left(x^{\prime \prime}, y\right) \\
& \quad=\sum_{i=1}^{m-2} c_{i, 0}^{\prime \prime}\left(x^{\prime \prime}\right)^{i}+\left(a_{m, 0}+c_{m, 0}^{\prime \prime}\right)\left(x^{\prime \prime}\right)^{m}+y\left(a_{01}^{\prime \prime}+c_{01}^{\prime \prime}\right) t^{p}+y^{-1}\left(a_{0,-1}^{\prime \prime}+c_{0,-1}^{\prime \prime}\right) t^{q}+\cdots,
\end{aligned}
$$

where we omit the monomials $\left(x^{\prime \prime}\right)^{i} y^{j}$ with $(i, j) \notin \Delta_{z}, \Delta_{z}=\operatorname{conv}\{(m, 0),(0,1),(0,-1)\}$, and we have

$$
c_{i, 0}^{\prime \prime}=c_{i, 0}^{\prime}+O(t)+\text { h.o.t. }, \quad i=0, \ldots, m-2, \quad c_{m, 0}^{\prime \prime}(0)=c_{01}^{\prime \prime}(0)=c_{0,-1}^{\prime \prime}(0)=0,
$$

whereas $a_{01}^{\prime \prime}, a_{0,-1}^{\prime \prime} \in \mathbb{C}^{*}$, and $p, q$ are distinct positive integers (we assume that $p<q$ ). By Lemma 3.10 the tropicalization of $f^{\prime \prime}\left(x^{\prime \prime}, y\right)$ determines a subdivision containing the triangle $\Delta_{z}$, and the corresponding deformation pattern. In particular, $c_{i, 0}^{\prime \prime}=O(t)$, $i=0, \ldots, m-2$. Another consequence is as follows: plugging the coordinates $x^{\prime \prime}=t \xi_{s}^{1}-\tau$, $y=\eta_{s}^{0}+\eta_{s}^{1} t$ of $\boldsymbol{p}_{s}$ in $f^{\prime \prime}\left(x^{\prime \prime}, y\right)$, we see that the minimal powers of $t$ come from the monomials $x^{m}$ and $y$, and they must compensate each other, because the coordinates of $\boldsymbol{p}_{s}$ annihilate $f^{\prime \prime}$, i.e.,

$$
\eta_{s}^{0} a_{01}^{\prime \prime} t^{p}+a_{m, 0}\left(\xi_{s}^{1} t-\tau\right)^{m}+\text { h.o.t. }=0 .
$$

This equation leads to

$$
\tau=\xi_{s}^{1} t-\left(-\frac{\eta_{s}^{o} a_{01}^{\prime \prime}}{a_{m, 0}}\right)^{1 / m} t^{p / m}+\text { h.o.t. }
$$


Combining this with the above formulas for $\tau$ and $c_{i, 0}^{\prime \prime}, c_{i, 0}^{\prime}$, it is not difficult to deduce the relation

$$
\begin{aligned}
c_{00}- & \frac{a_{00}}{a_{m, 0}} c_{m, 0}=c_{00}+(-1)^{m+1}\left(\xi_{s}^{0}\right)^{m} c_{m, 0} \\
\quad & =(-1)^{m} m a_{m, 0}\left(\xi_{s}^{0}\right)^{m-1} \xi_{s}^{1} t+(-1)^{m-1} m\left(\xi_{s}^{0}\right)^{m-1}\left(-\xi_{s}^{0} a_{01}^{\prime \prime} a_{m, 0}^{m-1}\right)^{1 / m} t^{p / m}+\Phi_{s},
\end{aligned}
$$

where $\Phi_{s}$ is some analytic function of the parameters $a_{i j}, c_{i j},(i, j) \in \Delta$, and $\xi_{s}^{0}, \xi_{s}^{1}, \eta_{s}^{0}$, $\eta_{s}^{1}, t$ whose terms contain $t$ to a positive power or $c_{i j}$ to the total power at least 2 . We point out that formula (5.4.26) gives $m$ distinct equations.

Thus, finally we obtain $\prod_{s=1}^{r}\left|\sigma_{s}\right|$ distinct systems of equations for the coefficients of $f(x, y)$. Each system consists of equations (5.4.24), (5.4.25), and (5.4.26); the latter equation is written as

$$
c_{\omega_{s}^{\prime}} a_{\omega_{s}^{\prime \prime}}-c_{\omega_{s}^{\prime \prime}} a_{\omega_{s}^{\prime}}=O(t)+\text { h.o.t., } \quad s=1, \ldots, r .
$$

Now we put $c_{i_{0} j_{0}}=0$ for some $\left(i_{0}, j_{0}\right) \in B$ and apply the implicit function theorem in order to conclude that the system has a unique solution. The conditions of the implicit function theorem are fulfilled because, for instance, the independence of the linearized system (5.4.25), (5.4.27) for $t=0$ is equivalent to that of system (3.7.13), (3.7.14) treated in Step 1 in Subsection 3.7 .

\section{$\S 6$. Counting Real nodal Curves}

Since complex conjugation naturally acts in $\mathbb{K}$, we can talk of real $\mathbb{K}$-curves, i.e., those defined over the subfield $\mathbb{K}_{\mathbb{R}}$ of Puiseux series with real coefficients. If, for instance, the given points $\boldsymbol{p}_{1}, \ldots, \boldsymbol{p}_{r}$ belong to $\left(\mathbb{K}_{\mathbb{R}}^{*}\right)^{2}$, then we can use the formulas of Lemmas 3.5, 3.6 and 3.9 to count how many real tropicalizations $(A, S, F, R) \in \mathcal{Q}_{\Delta}\left(n A_{1}\right)$ correspond to a nodal amoeba $A$. Then, taking the real solutions of equations (5.4.26), we can decide how many real nodal curves correspond to a given amoeba, and thereby confirm the formulas suggested by Mikhalkin in [18. Here we focus on a related problem of computing the Welschinger number $\chi_{\Delta}\left(\boldsymbol{p}_{1}, \ldots, \boldsymbol{p}_{r}\right)$, which was introduced in a general symplectic setting in 35. In our situation it is the number of real nodal irreducible curves passing through given real points and counted with the sign $(-1)^{n_{\text {iso }}}$, where, for a given real nodal curve, $n_{i s o}$ is the number of its real solitary nodes (i.e., those given locally by $\left.x^{2}+y^{2}=0\right)$. The importance of this number comes from Welschinger's theorem [35] saying that, for rational nodal curves, $\chi_{\Delta}\left(\boldsymbol{p}_{1}, \ldots, \boldsymbol{p}_{r}\right)$ does not depend on the choice of the fixed points. This means that, when calculated for a special configuration of $r=\left|\partial \Delta \cap \mathbb{Z}^{2}\right|-1$ real points, the number $\left|\chi_{\Delta}\left(\boldsymbol{p}_{1}, \ldots, \boldsymbol{p}_{r}\right)\right|$ provides a lower bound for the number of real rational curves passing through an arbitrary collection of $r$ generic real points in $\operatorname{Tor}(\Delta)$.

The next proposition is a consequence of the results of the preceding sections.

Proposition 6.1. In the notation of $\S 3$, for any generic points $\boldsymbol{x}_{1}, \ldots, \boldsymbol{x}_{r} \in \mathbb{Q}^{2}$ and $\boldsymbol{p}_{1}, \ldots, \boldsymbol{p}_{r} \in\left(\mathbb{K}_{\mathbb{R}}^{*}\right)^{2}$ such that $\operatorname{Val}\left(\boldsymbol{p}_{i}\right)=\boldsymbol{x}_{i}, i=1, \ldots, r$, and any irreducible nodal amoeba A of rank $r$ passing through $\boldsymbol{p}_{1}, \ldots, \boldsymbol{p}_{r}$, the following statements are true:

(i) if the dual subdivision $S$ contains an edge of even length, then the contribution to $\chi_{\Delta}\left(\boldsymbol{p}_{1}, \ldots, \boldsymbol{p}_{r}\right)$ of the real $n$-nodal curves passing through $\boldsymbol{p}_{1}, \ldots, \boldsymbol{p}_{r}$ and projecting to $A$ is zero;

(ii) if the dual subdivision $S$ has only edges of odd length, then there exists a unique real irreducible $n$-nodal curve passing through $\boldsymbol{p}_{1}, \ldots, \boldsymbol{p}_{r}$ and projecting to $A$, and its contribution to $\chi_{\Delta}\left(\boldsymbol{p}_{1}, \ldots, \boldsymbol{p}_{r}\right)$ is $(-1)^{s}$, where $s$ is the total number of interior integral points in the triangles of $S$. 
Proof. Recall that, by Lemma 3.7, to count the irreducible nodal curves we need to consider only irreducible nodal amoebas.

Suppose $S$ contains an edge $\sigma$ of even length $m$. The formulas of Lemma 3.9 show that given a real tropicalization $f_{1}, \ldots, f_{N}$, with the edge $\sigma$ we can associate either zero, or two real deformation patterns, which in their turn are independent of how many real solutions equations (5.4.26) have. If real deformation patterns do exist, their explicit formulas can be extracted from the computation in the proof of Lemma 3.9. Namely, one real deformation pattern corresponds to the Chebyshev polynomial $P(x)=$ $\cos \left(m \cdot \arccos \left(2^{-(m-1) / m} x\right)\right)$, and this deformation pattern has $m-1$ real solitary nodes by [22, Proposition 2.5]. The other real deformation pattern corresponds to the polynomial $-P(x \sqrt{-1})$, and it has one nonsolitary node besides $m-2$ imaginary nodes. Thus, claim (i) follows, because the exchange of the above deformation patterns changes the parity of the number of solitary real nodes.

If $S$ contains only edges of odd length, then the formulas in the proof of Lemmas 3.5 and 3.9 and equations (5.4.26) give a unique real choice for an $n$-nodal curve passing through $\boldsymbol{p}_{1}, \ldots, \boldsymbol{p}_{r}$ and projecting to $A$. It is a simple exercise to check that the real tropicalizations to triangles and the real deformation patterns associated with edges of odd length have only imaginary or real solitary nodes, whereas the real tropicalizations to parallelograms do not bear solitary real nodes. Thus, statement (ii) follows.

\section{REFERENCES}

[1] L. Caporaso and J. Harris, Counting plane curves of any genus, Invent. Math. 131 (1998), no. 2, 345-392. MR 1608583 (99i:14064)

[2] _ Parameter spaces for curves on surfaces and enumeration of rational curves, Compositio Math. 113 (1998), no. 2, 155-208. MR.1639183(99e:14061a)

[3] Xi Chen, Rational curves on K3 surfaces, J. Algebraic Geom. 8 (1999), 245-278. MR.1675158 (2000d:14057)

[4] L. Chiantini and C. Ciliberto, On the Severi varieties on surfaces in $\mathbf{P}^{3}$, J. Algebraic Geom. 8 (1999), no. 1, 67-83. MR1658208 (2000f:14082)

[5] S. Diaz and J. Harris, Ideals associated to deformations of singular plane curves, Trans. Amer. Math. Soc. 309 (1988), no. 2, 433-468. MR0961600 (89m:14003)

[6] M. Forsberg, M. Passare, and A. Tsikh, Laurent determinants and arrangements of hyperplane amoebas, Adv. Math. 151 (2000), 45-70. MR1752241 (2001m:32060)

[7] I. M. Gel'fand, M. M. Kapranov, and A. V. Zelevinsky, Discriminants, resultants, and multidimensional determinants, Birkhäuser Boston, Inc., Boston, MA, 1994. MR1264417 (95e:14045)

[8] A. Henriques, An analogue of convexity for complements of amoebas of varieties of higher codimensions, Preprint, Berkeley, May 2001.

[9] A. Hirschowitz, La méthode d'Horace pour l'interpolation à plusieurs variables, Manuscripta Math. 50 (1985), 337-388. MR0784148 (86j:14013)

[10] I. Itenberg and E. Shustin, Singular points and limit cycles of planar polynomial vector fields, Duke Math. J. 102 (2000), no. 1, 1-37. MR1741776 (2001d:34019)

[11] - Viro theorem and topology of real and complex combinatorial hypersurfaces, Israel J. Math. 133 (2003), 189-238. MR.1968429 (2004c:14115)

[12] M. M. Kapranov, Amoebas over non-Archimedean fields, Preprint, 2000.

[13] M. Kontsevich and Y. Soibelman, Homological mirror symmetry and torus fibrations, Preprint arXiv:math.SG/0011041.

[14] M. Kontsevich and Yu. Tschinkel, Non-Archimedean Kähler geometry (in preparation).

[15] G. Mikhalkin, Real algebraic curves, the moment map and amoebas, Ann. of Math. (2) 151 (2000), no. 1, 309-326. MR.1745011 (2001c:14083)

[16] _ Amoebas of algebraic varieties, Preprint arXiv:math.AG/0108225.

[17] G. Mikhalkin and H. Rullgård, Amoebas of maximal area, Internat. Math. Res. Notices 2001, no. 9, 441-451. MR:1829380 (2002b:14079)

[18] G. Mikhalkin, Counting curves via lattice paths in polygons, C. R. Math. Acad. Sci. Paris 336 (2003), no. 8, 629-634. MR1988122 (2004d:14077)

[19] _ Enumerative tropical algebraic geometry in $\mathbb{R}^{2}$, Preprint arXiv: math AG/0312530. 
[20] M. Passare and H. Rullgård, Amoebas, Monge-Ampère measures and triangulations of the Newton polytope, Res. Rep. in Math. no. 10, Stockholm Univ., 2000.

[21] H. Rullgård, Stratification des espaces de polynômes de Laurent et la structure de leurs amibes, C. R. Acad. Sci. Paris Sér. I Math. 331 (2000), 355-358. MR.1784913 (2001h:32044)

[22] E. Shustin, Real plane algebraic curves with prescribed singularities, Topology 32 (1993), 845-856. MR.1241875 (95f:14049)

[23] - Surjectivity of the resultant map: A solution to the inverse Bezout problem, Comm. Algebra 23 (1995), no. 3, 1145-1163. MR1316754 (96a:12004)

[24] _ Critical points of real polynomials, subdivisions of Newton polyhedra and topology of real algebraic hypersurfaces, Topology of Real Algebraic Varieties and Related Topics, Amer. Math. Soc. Transl. (2), vol. 173, Amer. Math. Soc., Providence, RI, 1996, pp. 203-223. MR1384319)(97g:14040)

[25] _ Gluing of singular and critical points, Topology 37 (1998), no. 1, 195-217. MR1480886 (99f:14073)

[26] - Lower deformations of isolated hypersurface singularities, Algebra i Analiz 11 (1999), no. 5, 221-249; English transl., St. Petersburg Math. J. 11 (2000), no. 5, 883-908. MR.1734355 (2000m:32039)

[27] _ Patchworking singular algebraic curves, non-Archimedean amoebas and enumerative geometry, Preprint arXiv: math.AG/0211278.

[28] E. Shustin and I. Tyomkin, Patchworking singular algebraic curves, to appear in Israel J. Math.

[29] B. Sturmfels, Solving systems of polynomial equations, CBMS Regional Conf. Ser. in Math., vol. 97, Amer. Math. Soc., Providence, RI, 2002. MR/1925796 (2003i:13037)

[30] O. Ya. Viro, Gluing of algebraic hypersurfaces, smoothing of singularities and construction of curves, Proceedings of the Leningrad International Topological Conference (Aug. 1982), "Nauka", Leningrad, 1983, pp. 149-197. (Russian)

[31] _ Gluing of plane real algebraic curves and constructions of curves of degrees 6 and 7, Topology (Leningrad, 1982), Lecture Notes in Math., vol. 1060, Springer, Berlin, 1984, pp. 187200. MR0770238 (87i:14029)

[32] Real algebraic plane curves: Constructions with controlled topology, Algebra i Analiz 1 (1989), no. 5, 1-73; English transl., St. Petersburg Math. J. 1 (1990), no. 5, 1059-1134. MR1036837 (91b:14078)

[33] , Patchworking real algebraic varieties, Preprint at http://www.math.uu.se/ oleg/preprints. html (1995).

[34] J. Wahl, Equisingular deformations of plane algebraic curves, Trans. Amer. Math. Soc. 193 (1974), 143-170. MR0419439 (54:7460)

[35] J.-Y. Welschinger, Invariants of real rational symplectic 4-manifolds and lower bounds in real enumerative geometry, C. R. Math. Acad. Sci. Paris 336 (2003), 341-344. MR1976315 (2004m:53157)

School of Mathematical Sciences, Tel Aviv University, Ramat Aviv, Tel Aviv 69978, Israel E-mail address: shustin@post.tau.ac.il

Received 20/JUN/2003

Originally published in English 